Electronic Supporting Information

for the manuscript entitled

\title{
Chirality Inversion of CdSe and CdS Quantum Dots Without Changing Stereochemistry of the Capping Ligand
}

Jung Kyu Choi, ${ }^{a}$ Benjamin E. Haynie, ${ }^{a}$ Urice Tohgha, ${ }^{a}$ Levente Pap, ${ }^{a}$ K. Wade Elliott, ${ }^{a}$ Brian M.

Leonard, ${ }^{\text {a }}$ Sergei V. Dzyuba, ${ }^{\mathrm{b}}$ Krisztina Varga, ${ }^{* a}$ Jan Kubelka, ${ }^{* a}$ and Milan Balaz*c

(a) University of Wyoming, Department of Chemistry, Laramie, WY 82071, USA.

(b) Texas Christian University, Department of Chemistry and Biochemistry, Fort Worth, TX 76129, USA.

(c) Yonsei University, Underwood International College, Integrated Science \& Engineering Division, Seoul, 03722, Republic of Korea. 


\section{TABLE OF CONTENTS}

Materials and Methods

... S2-S4

Figure S1: CD spectrum of $N$-Ac-L-Cys-Me-ester-CdSe QDs $\left(\varnothing_{\mathrm{CdSe}}=4.4 \mathrm{~nm}\right) \quad \ldots \quad \mathrm{S} 4$

Figure S2: CD and UV-vis spectra CdSe QDs $\left(\varnothing_{\mathrm{CdSe}}=2.9 \mathrm{~nm}\right)$ functionalized with $\quad \ldots$ S4 cysteine and its derivatives

Figure S3: CD, UV-vis and FDCD spectra of L-Cys-CdS and $\quad \ldots$ S5 $N$-Ac-L-Cys-CdS $\left(\varnothing_{\mathrm{CdS}}=3.7 \mathrm{~nm}\right)$

Figure S4: Variable concentration CD and UV-vis absorption spectra of L-Cys-CdS $\quad \ldots \quad$ S5 $\left(\varnothing_{\mathrm{CdS}}=4.3 \mathrm{~nm}\right)$ and $N$-Ac-L-Cys-CdS $\left(\varnothing_{\mathrm{CdS}}=4.3 \mathrm{~nm}\right)$

Figures S5-S6: HRTEM images of cysteine derivative functionalized CdSe and CdS _.. S6

Figure S7-S8: Powder XRD patterns of cysteine derivative functionalized CdSe and CdS $\ldots$ S7

Figure S9: CD spectra of $N$-Ac-L-Cys-CdSe $\left(\varnothing_{\mathrm{CdSe}}=4.4 \mathrm{~nm}\right)$, a precipitation series $\ldots$ S8

Figure S10: Model of CdSe QD generated using CrystalMaker software $\quad \ldots \quad$ S8

Figures S11-S15: TGA curves for $N$-Ac-L-Cys-CdSe $\left(\varnothing_{\mathrm{CdSe}}=4.4 \mathrm{~nm}\right) \quad \ldots \quad$ S8-S10

Table S1: Ligand-to-surface ratios $\quad \ldots$ S10

Table S2: CD anisotropy $g$ factors $\quad \ldots$ S10

Figure S16: $1 \mathrm{D}{ }^{13} \mathrm{C}$ MAS ssNMR spectra of cysteine derivatives and cysteine $\quad \ldots$ S11 derivative functionalized QDs $\left(\varnothing_{\mathrm{CdSe}}=4.4 \mathrm{~nm}\right)$

Figure S17: FTIR spectra of cysteine ligands and cysteine derivative functionalized CdSe $\ldots$. S12

$\begin{array}{lll}\text { Computational methods } & \ldots & \mathrm{S} 13\end{array}$

Figure S18: Model CdSe quantum dot fragment and simulated UV absorption spectrum $\quad \ldots \quad$ S13

Figure S19: Tri-dentate binding of $N$-Ac-L-Cys to the QD surface $\quad \ldots$ S14

Table S3: Relative energies in kcal.mol ${ }^{-1}$ of CdSe complexes with a single ligand $\quad \ldots$ S15

Table S4: Relative energies in kcal.mol ${ }^{-1}$ of CdSe complexes with two ligands $\quad \ldots$ S15

Figure S20: Simulated UV and CD spectra for model CdSe QD with a single ligand _.. S16

Figure S21-S24: Schematic illustrations of the bi-dentate binding arrangements $\quad \ldots \quad$ S17-S20 on the model QD surfaces, constructed as repeats of the elementary CdSe fragment used as a minimal model

Figure S25-S29: Simulated UV and CD spectra for model CdSe QD with two

.. S21-S25

$N$-Ac-L-Cys ligands or two L-HomoCys ligands

Coordinates: $\mathrm{Cd}_{9} \mathrm{Se}_{7} \mathrm{H}_{22}$ cluster $\quad \ldots \quad \mathrm{S} 26$

$\begin{array}{lll}\text { References } & \ldots \text { S27 }\end{array}$ 


\section{Materials}

L-cysteine.HCl (L-Cys), D-cysteine (D-Cys), $N$-acetyl-L-cysteine ( $N$-Ac-L-Cys), L-cysteine methyl ester.HCl (L-Cys-Me-ester), L-penicillamine (L-Pen), $N$-acetyl-L-cysteine methyl ester ( $N$-Ac-L-Cys-Meester), and tetramethyl-ammonium hydroxide (TMAH) were purchased from Sigma-Aldrich. Lhomocysteine (L-HomoCys), $N$-acetyl-D-cysteine ( $N$-Ac-D-Cys), D-cysteine (D-Cys), and DPenicillamine (D-Pen) were purchased from P212121, LLC. Oleic acid functionalized CdS QDs (OACdS) were purchased from NN-labs, LLC. All commercial chemicals were used without further purification. Water was obtained from a Milli-Q system with a resistivity of $18.2 \mathrm{M} \Omega \bullet \mathrm{cm}$.

\section{Methods}

Oleic acid functionalized $\mathrm{CdSe}$ (OA-CdSe) QDs were synthesized following a previously reported procedure, ${ }^{1,2}$ and their diameter was determined using Peng's equation from the absorption spectra. ${ }^{3}$

Synthesis of CdSe functionalized with cysteine derivatives from OA-CdSe QDs by phase transfer ligand exchange: ${ }^{1}$ Cysteine derivative was dissolved in DI water $(20 \mathrm{~mL}$, [L-Cys] $=0.056 \mathrm{M})$. The $\mathrm{pH}$ of the resulting solution was adjusted to 12.0 with tetramethylammonium hydroxide. A solution of OA-CdSe QDs in toluene $\left(12 \mathrm{~mL}, 0.03 \mathrm{mM}, \mathrm{A}_{\mathrm{exc}}=0.85\right)$ was added to the cysteine solution and the reaction mixture was deoxygenated. The reaction mixture was stirred at room temperature under $\mathrm{N}_{2}$ in the absence of light for $24 \mathrm{~h}$. The reaction mixture was allowed to stand without stirring until the phases fully separated. The aqueous layer was withdrawn using a syringe, and the CdSe QDs capped with cysteine derivatives were precipitated by addition of acetone to the toluene solution, centrifuged and separated from the colorless supernatant. The precipitated CdSe QDs capped with cysteine derivatives were redissolved in $\mathrm{DI} \mathrm{H}_{2} \mathrm{O}(2 \mathrm{~mL})$ and were stored in the dark.

Synthesis of CdS functionalized with cysteine derivatives from OA-CdSe QDs by phase transfer ligand exchange: ${ }^{1}$ CdS QDs $(\varnothing=4.3 \mathrm{~nm}$ and $3.6 \mathrm{~nm})$ functionalized with cysteine derivatives (L-Cys and $N$-AcL-Cys) were synthesized from OA-CdS QDs. An aqueous solution of the cysteine derivative ( $5 \mathrm{~mL}, 0.057$ $\mathrm{M}, \mathrm{pH}$ 12) was added to a toluene solution of OA-CdS QDs ( $\left.3 \mathrm{~mL}, \mathrm{~A}_{\mathrm{exc}}=0.85\right)$ in a $25 \mathrm{~mL}$ flask, and the $\mathrm{pH}$ was adjusted to 12 with tetramethylammonium hydroxide. The resulting mixture was deoxygenated under vigorous stirring using several vacuum- $\mathrm{N}_{2}$ cycles, followed by stirring at RT under $\mathrm{N}_{2}$ in the absence of light for $24 \mathrm{~h}$. The reaction mixture was left to stand until the phases fully separated. The aqueous layer was removed with a syringe, and the CdSe QDs capped with cysteine derivatives were precipitated by addition of acetone to the toluene solution, centrifuged and separated from the colorless supernatant. The CdS QDs capped with cysteine derivative were then re-dissolved in $\mathrm{DI}_{2} \mathrm{O}(2 \mathrm{~mL})$ and stored in the dark.

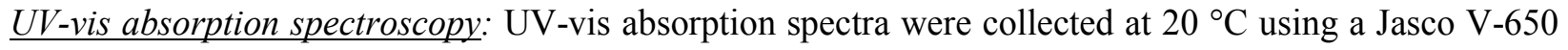
UV-vis double beam spectrophotometer equipped with a single position Peltier temperature control system, using a $1 \mathrm{~cm}$ path length quartz cuvette.

Fluorescence spectroscopy: Emission measurements were carried out at $20{ }^{\circ} \mathrm{C}$ using a Varian Eclipse fluorescence spectrophotometer or a Jasco FP-8300 fluorescence spectrometer equipped with Peltier temperature control systems, using a $1 \mathrm{~cm}$ path length quartz cuvette. Scanning conditions: scan rate of $600 \mathrm{~nm} / \mathrm{min}$, excitation wavelength $450 \mathrm{~nm}$, with $5.0 \mathrm{~nm}$ excitation slit, and $5.0 \mathrm{~nm}$ emission slit.

Circular dichroism (CD) spectroscopy: CD spectra were recorded at $20{ }^{\circ} \mathrm{C}$ using a Jasco J-815 spectropolarimeter equipped with a Peltier temperature control system. Conditions were as follows: 
scanning speed $100 \mathrm{~nm} / \mathrm{min}$, data pitch $0.5 \mathrm{~nm}$, DIT $1 \mathrm{~s}$, and bandwidth $4 \mathrm{~nm}$. Each CD spectrum was an average of at least fifteen scans. All spectra were background subtracted and smoothed using manufacturer provided software Spectra Manager. Quartz cuvettes with a 0.1 or $1 \mathrm{~cm}$ path length were used for CD experiments.

Fluorescence detected circular dichroism (FDCD) spectroscopy: FDCD spectra were recorded at $20{ }^{\circ} \mathrm{C}$ using a Jasco J-815 spectropolarimeter equipped with a single position FDCD Peltier temperature control system and a FDCD-465 attachment combining both a cylindrical cell and an elliptical cylinder mirror. Conditions were as follows: scanning speed $100 \mathrm{~nm} / \mathrm{min}$, data pitch $0.5 \mathrm{~nm}$, DIT $1 \mathrm{~s}$, bandwidth $6 \mathrm{~nm}$, $580 \mathrm{~nm}$ filter, and masks \#7. Each FDCD spectrum was an average of at least six scans.

Magic angle spinning solid state Nuclear Magnetic Resonance (MAS ssNMR): MAS ssNMR experiments were carried out on a $600 \mathrm{MHz}$ Avance III Bruker NMR spectrometer equipped with a $3.2 \mathrm{~mm} \mathrm{E}^{\text {free }}$ triple resonance HCN probe. Samples of chiral QDs were packed into $4 \mathrm{~mm}$ Bruker rotors. To prepare the ssNMR sample, CdSe QDs functionalized with cysteine derivatives were lyophilized overnight from water. 1D ${ }^{13} \mathrm{C}$ ssNMR spectra were acquired using ${ }^{1} \mathrm{H}-{ }^{13} \mathrm{C}$ cross-polarization $(\mathrm{CP})$ and ${ }^{1} \mathrm{H}$ decoupling during acquisition. For the CdSe QDs, ${ }^{1} \mathrm{H}^{13} \mathrm{C} \mathrm{CP}$ was achieved with $70-100 \%$ ramped $63 \mathrm{kHz}{ }^{1} \mathrm{H}$ and 50 $\mathrm{kHz}{ }^{13} \mathrm{C}$ pulses for $2.0 \mathrm{~ms}$. Spectra were acquired for $20-40 \mathrm{~ms}$ with $78 \mathrm{kHz}$ of two-pulse phase modulated (TPPM) ${ }^{1} \mathrm{H}$ decoupling. Experiments were performed at $26{ }^{\circ} \mathrm{C}$ (variable temperature set point) and $8.0 \mathrm{kHz}$ MAS frequency. All spectra were externally referenced to DSS using the adamantane downfield ${ }^{13} \mathrm{C}$ peak at 40.48 ppm. ${ }^{4}$ Data were processed with Bruker's TopSpin software package.

Fourier transform infrared spectroscopy (FTIR): FTIR spectra were acquired at RT on Bruker Alpha FTIR with ATR Sampling Module and processed using OPUS 7 Spectroscopy Software.

Transmission electron microscopy (TEM): Imaging was performed on an FEI Tecnai G2 F20 scanning transmission electron microscope (STEM) operating at $200 \mathrm{kV}$. Samples for TEM were prepared by ultrasonic dispersion of the CdSe QDs in water. The suspension was then drop-cast onto carbon-coated copper grids and dried in air.

$\underline{X \text {-ray diffraction (XRD): }}$ Powder XRD data were collected on a Bruker - Axs Smart Apex II CCD Diffractometer.

Thermogravimetric analysis (TGA): Samples for TGA were prepared by lyophilizing aqueous solutions of CdSe QDs functionalized by cysteine derivative. TGA data were collected on a Mettler-Toledo thermogravimetric analyzer (heating rate: $10^{\circ} \mathrm{C} / \mathrm{min}$ ) under a nitrogen atmosphere ( 0.5-1.0 $\mathrm{mg}$ sample; flow rate $50 \mathrm{ml} / \mathrm{min}$ ). All experiments were carried out in triplicate.

Determination of the ligand-to-surface ratio: TGA was used to determine the amount of ligands (bound and unbound) and CdSe residues in the lyophilized QDs samples (Figure S11-S15). The ligand amounts were varied by a series of precipitations of aqueous solution of $\mathrm{N}$-Ac-L-Cys-CdSe with acetone (from Ligand:Cd of 8 to 0.78 ; Table S1) or by size exclusion chromatography (SEC, to L:Cd of 0.54). Attempts to further decrease the amount of capping ligands by precipitations let to insoluble material. The number of available cadmium binding sites on the CdSe surface $\left(\varnothing_{\mathrm{CdSe}}=4.4 \mathrm{~nm}\right)$ was estimated to be around 300 using CrystalMaker software (Figure S10). 


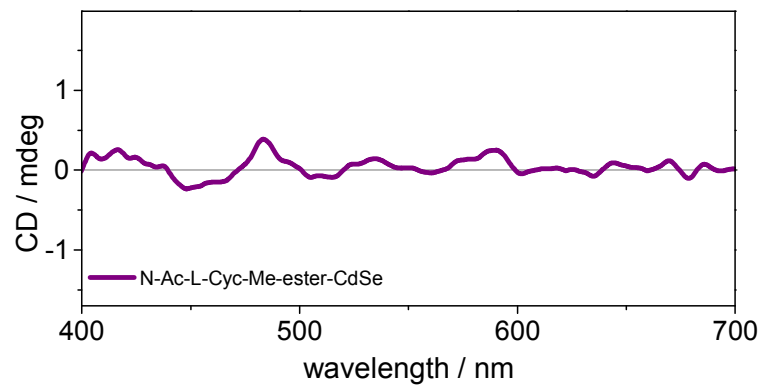

Figure S1. CD spectrum of CdSe QDs $\left(\varnothing_{\mathrm{CdSe}}=4.4 \mathrm{~nm}\right)$ functionalized with $N$-Ac-L-Cys-Me-ester. Conditions: $[\mathrm{CdSe}]=2.8 \mu \mathrm{M}$.

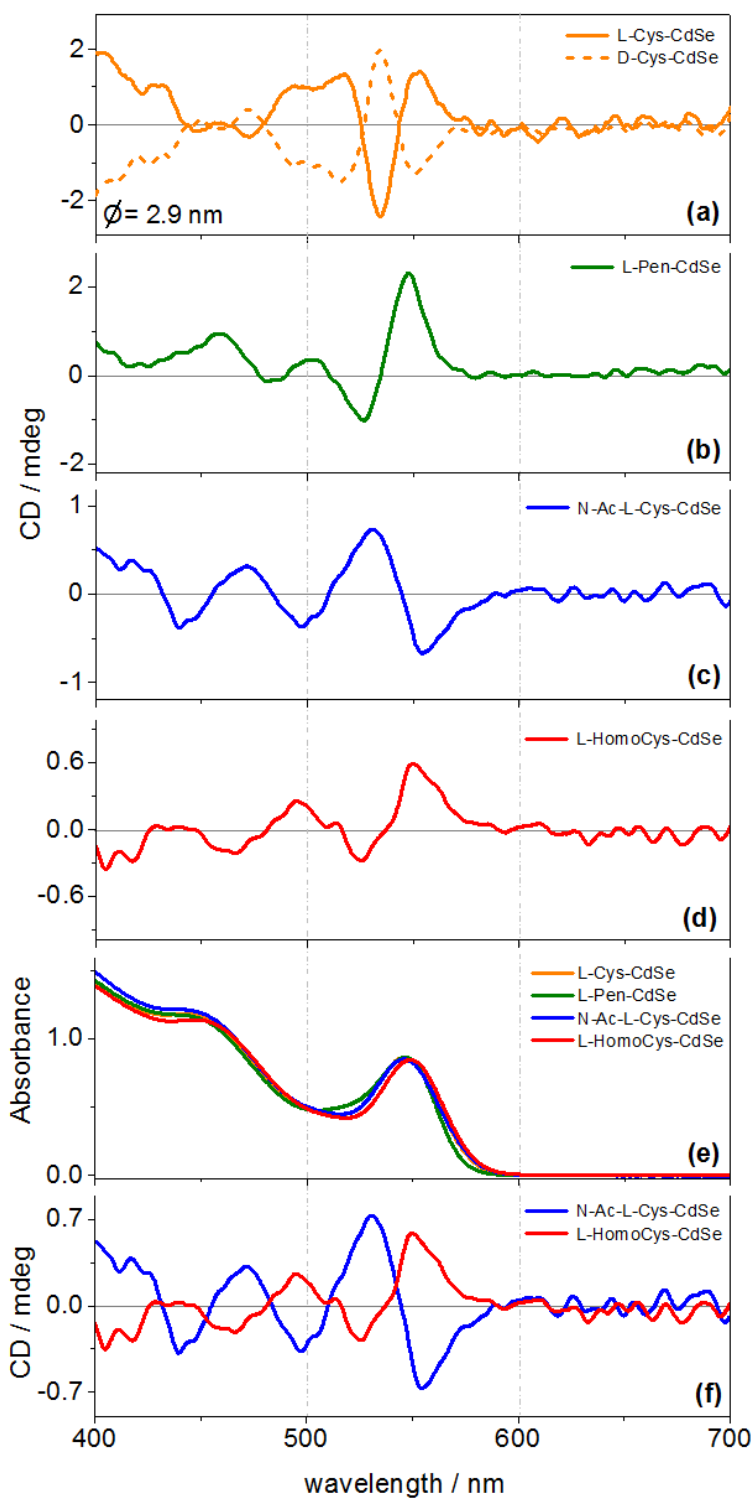

Figure S2. (a-d) CD and (e) UV-vis absorption spectra of CdSe QDs $\left(\varnothing_{\mathrm{CdSe}}=2.9 \mathrm{~nm}\right)$ functionalized with (a) L-Cys and D-Cys, (b) L-Pen, (c) N-Ac-L-Cys, and (d) L-HomoCys capping ligands. (f) 'Mirrorimage' CD spectra of L-HomoCys-CdSe and $N$-Ac-L-Cys-CdSe. $[\mathrm{CdSe}]=8.5 \mu \mathrm{M}\left(\varnothing_{\mathrm{CdSe}}=2.9 \mathrm{~nm}\right)$. 


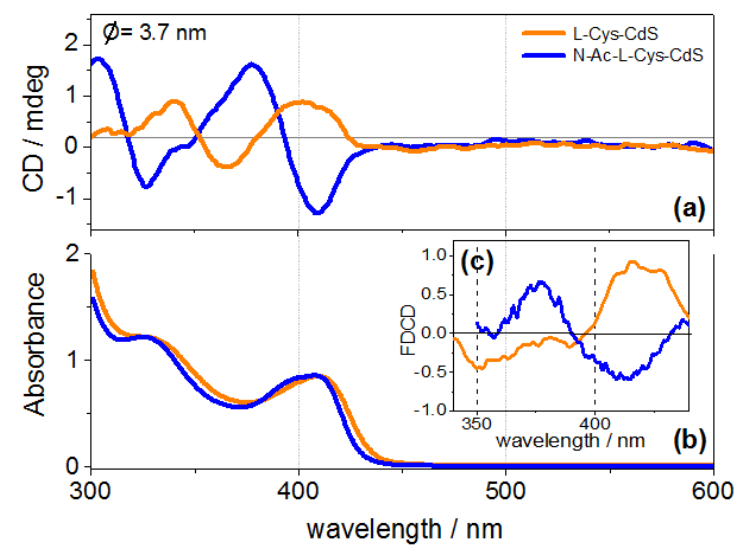

Figure S3. (a) CD, (b) UV-vis absorption, and (c) FDCD spectra of CdS QDs $\left(\varnothing_{\mathrm{CdS}}=3.7 \mathrm{~nm}\right)$ functionalized with L-Cys and $N$-Ac-L-Cys capping ligands. $[\mathrm{CdS}]=1.3 \mu \mathrm{M}$.
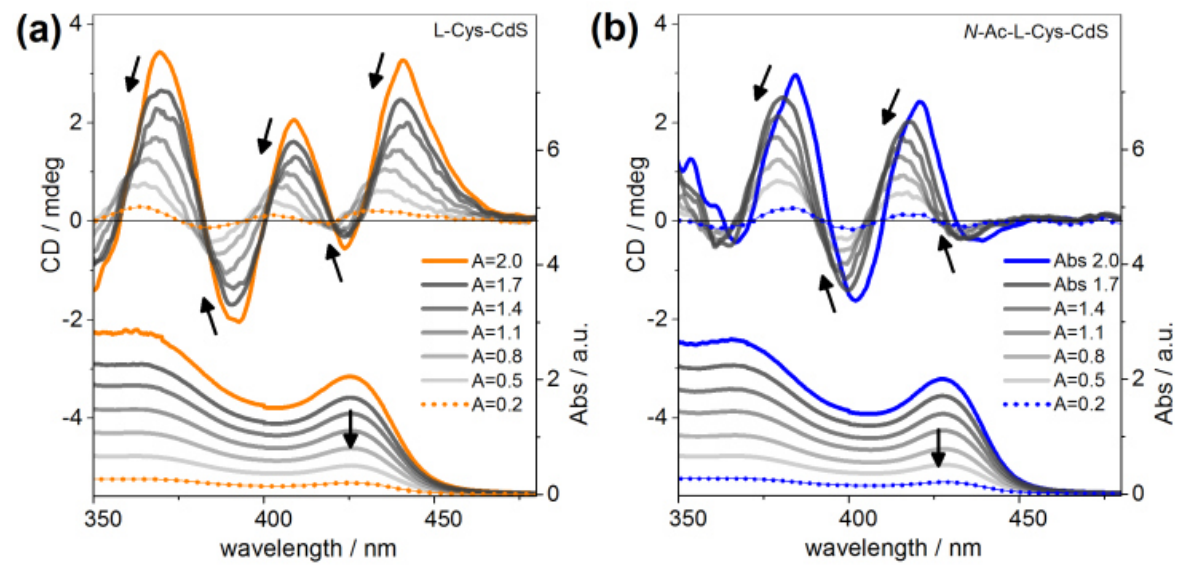

Figure S4. (a) Variable concentration CD and UV-vis spectra of L-Cys-CdS ( $\varnothing_{\mathrm{CdS}}=4.3 \mathrm{~nm}$ ). (b) Variable concentration $\mathrm{CD}$ and UV-vis spectra of $N$-Ac-L-Cys-CdS $\left([\mathrm{CdS}]=\right.$ from $3.2 \times 10^{-6} \mathrm{M}$ to $\left.0.32 \times 10^{-6} \mathrm{M} ; \varnothing_{\mathrm{CdS}}=4.3 \mathrm{~nm}\right)$. 

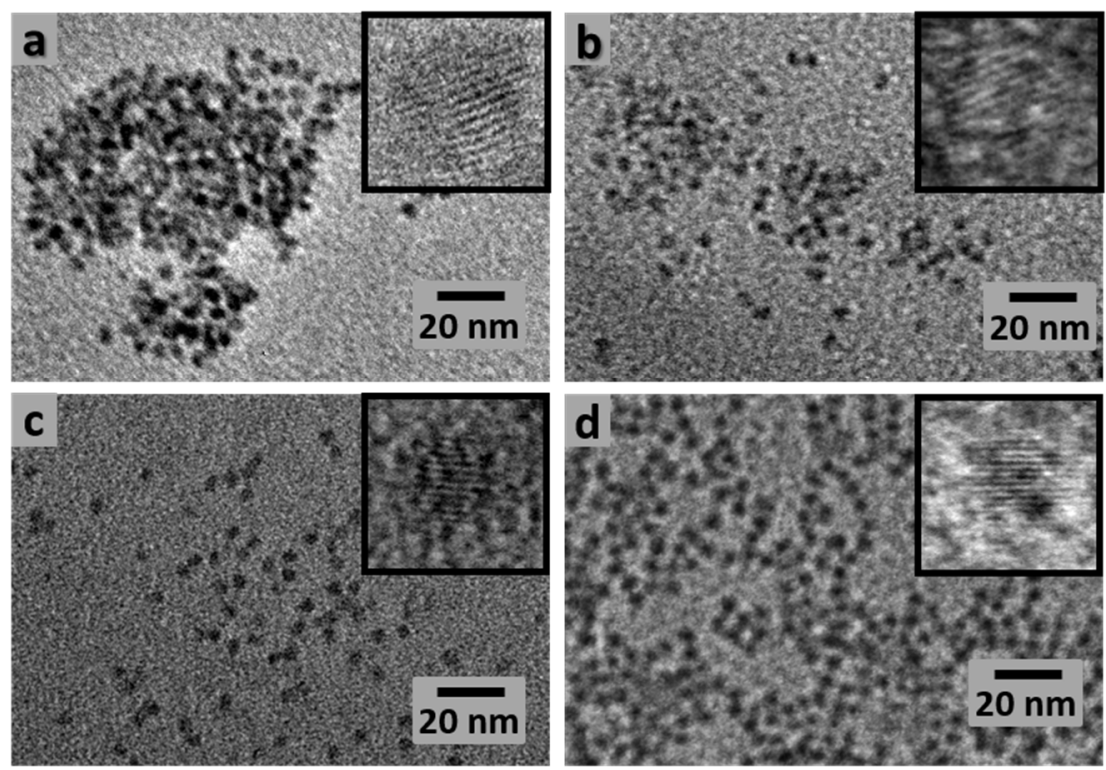

Figure S5. HRTEM images of (a) L-Cys-CdSe, (b) L-Cys-Me-ester-CdSe, (c) L-Pen-CdSe, (d) N-Ac-LCys-Me-ester-CdSe $\left(\varnothing_{\mathrm{CdSe}}=4.4 \mathrm{~nm}\right)$. Insets: Images of QDs showing a 111 lattice spacing of $\sim 3.48 \AA$ (box size: $5 \times 5 \mathrm{~nm}$ ).

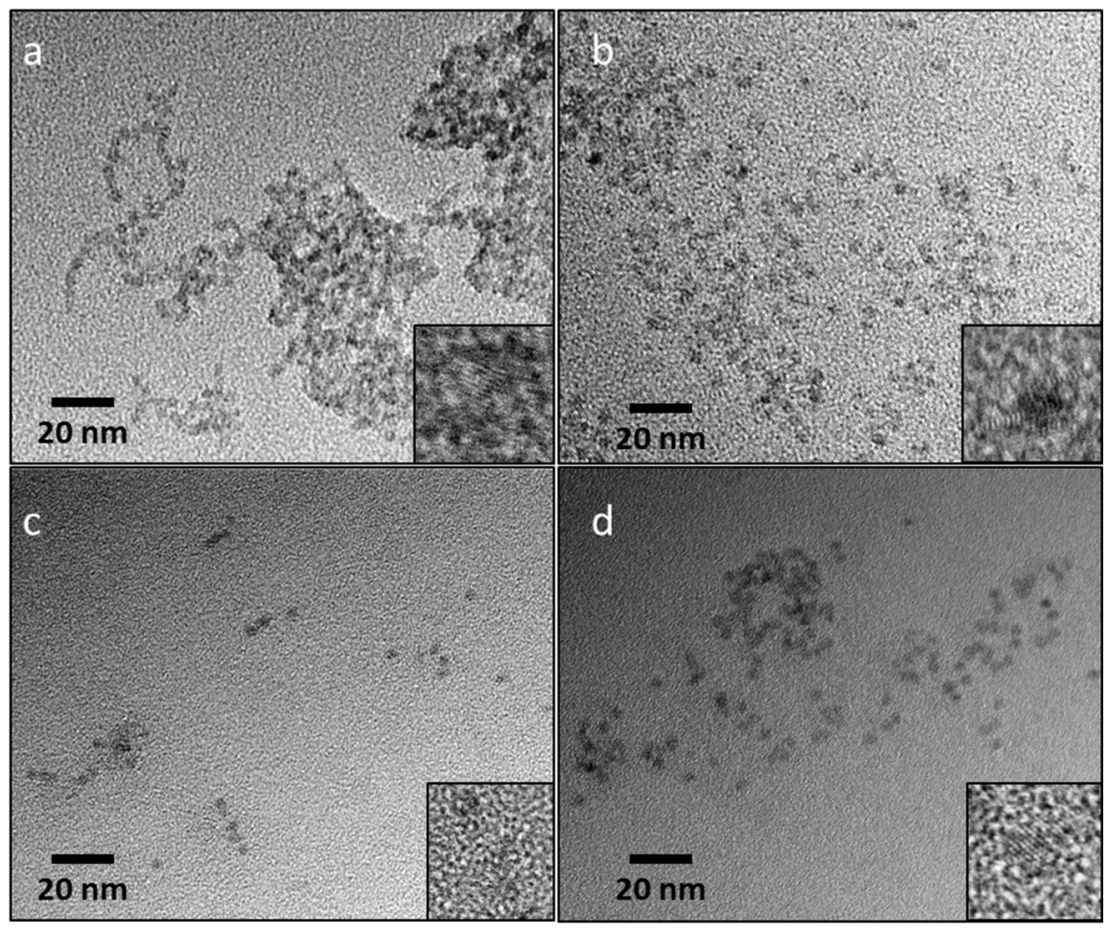

Figure S6. HRTEM images of (a) D-Cys-CdS, (b) N-Ac-D-Cys-CdS, (c) L-Cys-CdS, (d) N-Ac-L-Cys$\mathrm{CdS}\left(\varnothing_{\mathrm{CdS}}=4.3 \mathrm{~nm}\right)$. Insets: Images of QDs showing a 111 lattice spacing of $\sim 3.35 \AA$ (box size: $5 \times 5 \mathrm{~nm}$ ). 

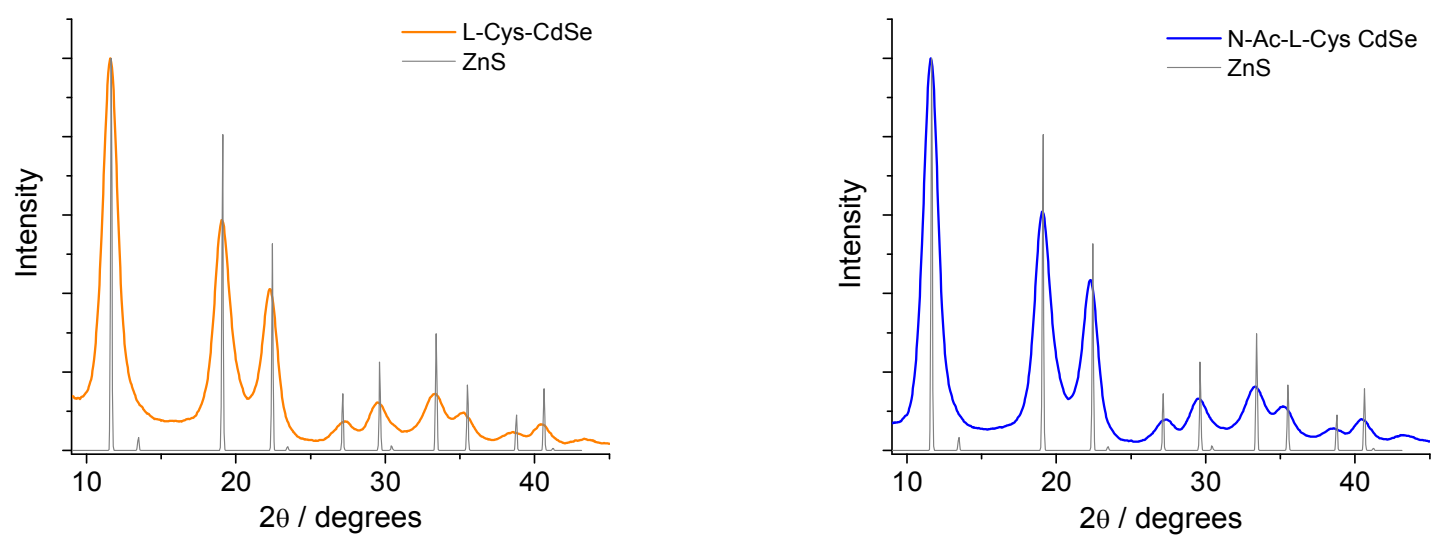

Figure S7. Experimental powder XRD patterns of L-Cys-CdSe QDs (left, $\varnothing=4.4 \mathrm{~nm}$ ) and $N$-Ac-L-CysCdSe QDs (right, $\varnothing=4.4 \mathrm{~nm}$ ) and XRD pattern of $\mathrm{ZnS}$ (cubic) reported in the ICDD powder diffraction files (No. 077-2100, grey lines).
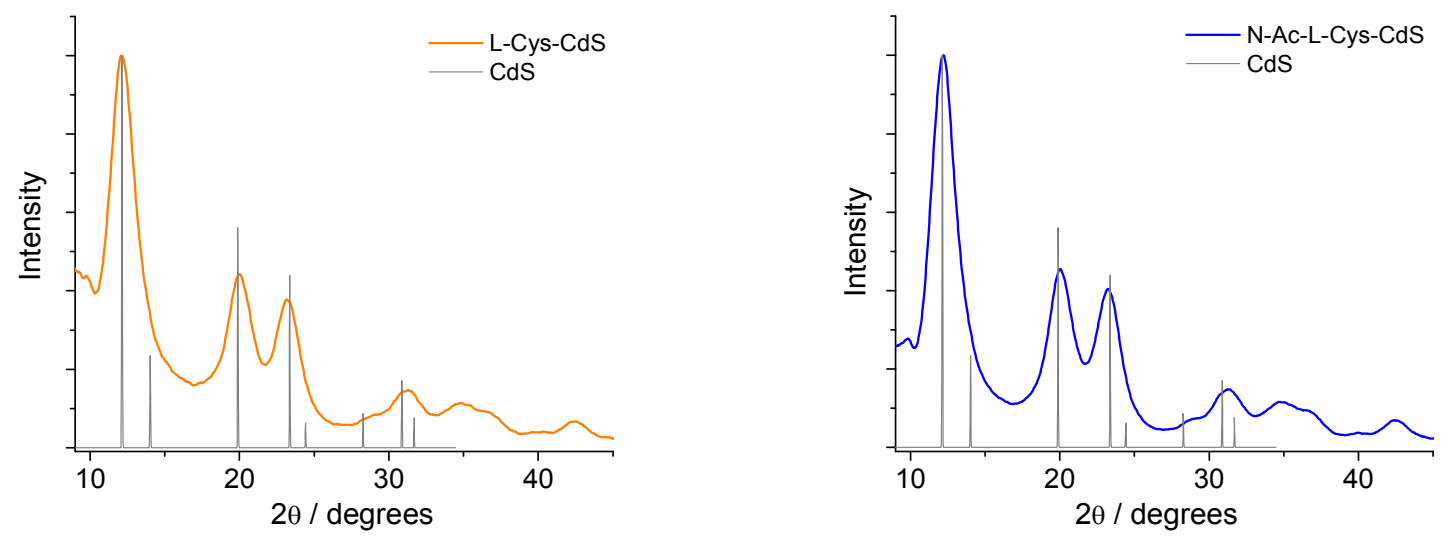

Figure S8. Observed powder XRD patterns of L-Cys-CdS QDs (left, $\varnothing=4.5 \mathrm{~nm}$ ) and $N$-Ac-L-Cys-CdS QDs (right, $\varnothing=4.5 \mathrm{~nm}$ ) with XRD pattern of $\mathrm{ZnS}$ (cubic) reported in the ICDD powder diffraction files (No. 075-0581, grey lines). 

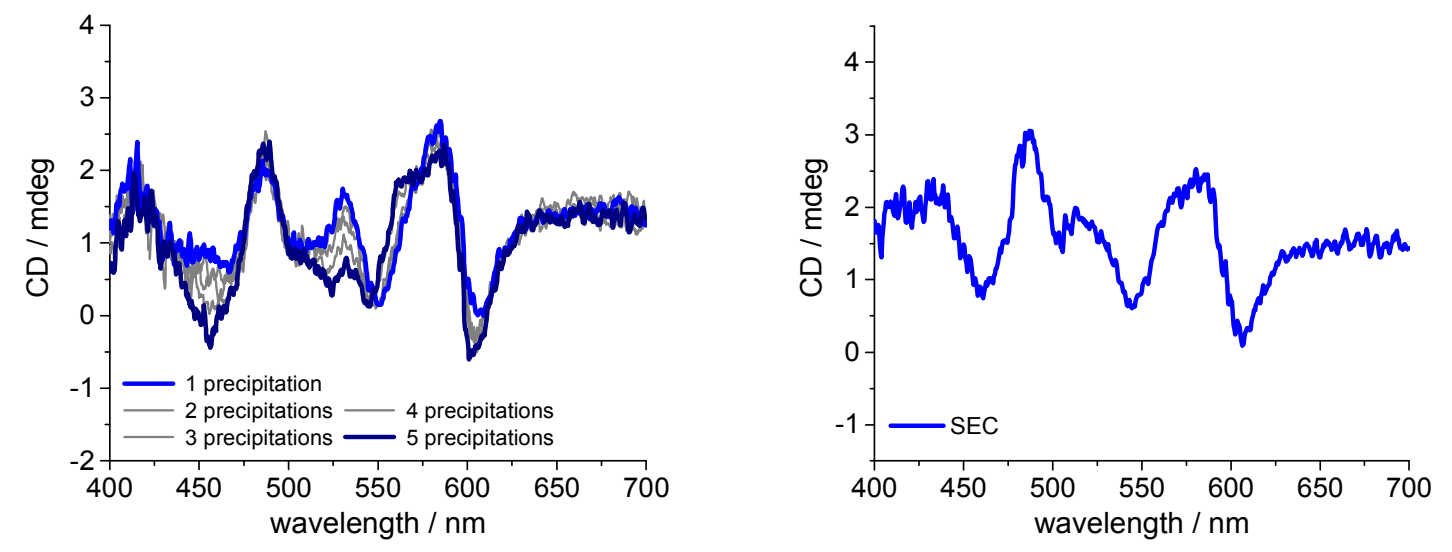

Figure S9. Left: Unsubtracted CD spectra of $N$-Ac-L-Cys-CdSe QDs $\left(\varnothing_{\mathrm{CdSe}}=4.4 \mathrm{~nm}\right)$ after a series of precipitations. Right: Unsubtracted CD spectrum of $N$-Ac-L-Cys-CdSe QDs $\left(\varnothing_{\mathrm{CdSe}}=4.4 \mathrm{~nm}\right)$ after purification by a size exclusion chromatography (SEC) of the crude material. $[\mathrm{CdSe}]=2.8 \mu \mathrm{M}$.

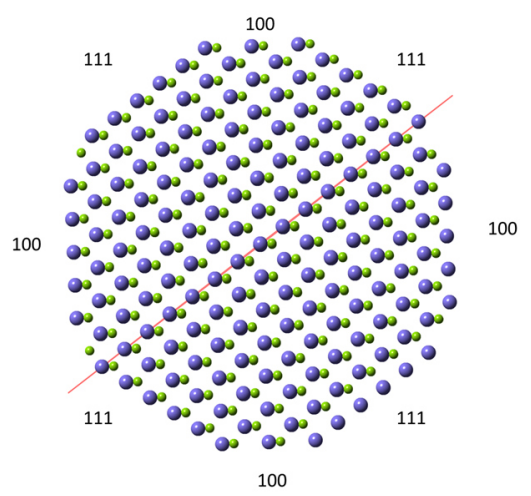

Figure S10. Model of CdSe QD generated using CrystalMaker software.

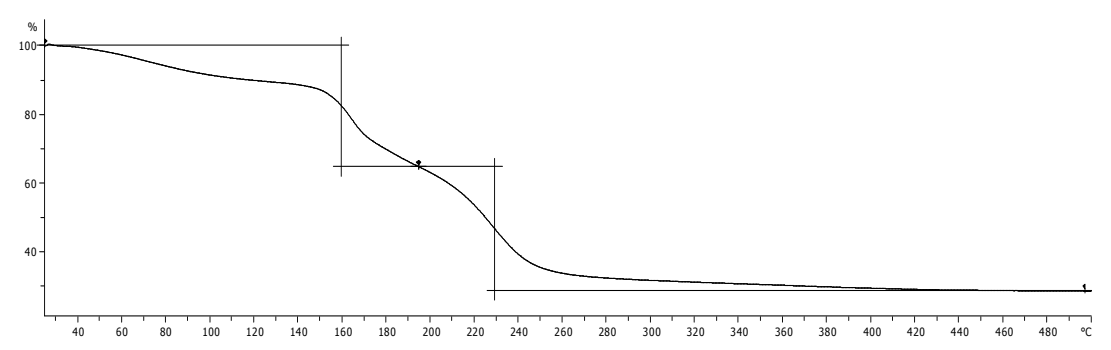

Figure S11. TGA data for $N$-Ac-L-Cys-CdSe (purified by 2 precipitations with acetone, $\varnothing_{\mathrm{CdSe}}=4.4 \mathrm{~nm}$ ) under $\mathrm{N}_{2}$ atmosphere. TGA curve shows weight loss of water below $130{ }^{\circ} \mathrm{C}$ and weight loss of $\mathrm{N}$-Ac-LCys capping ligand $\left(59.6 \mathrm{wt} \%\right.$ ) between $\sim 150{ }^{\circ} \mathrm{C}$ and $460{ }^{\circ} \mathrm{C}$. 


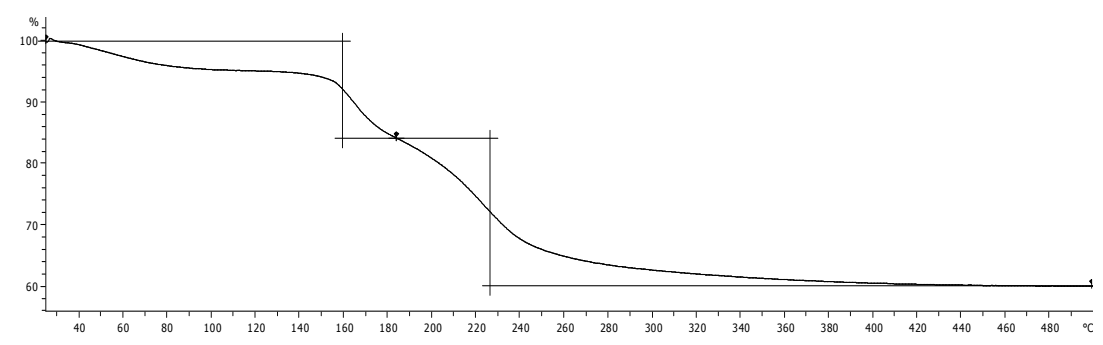

Figure S12. TGA data for $N$-Ac-L-Cys-CdSe (purified by 3 precipitations with acetone, $\varnothing_{\text {CdSe }}=4.4 \mathrm{~nm}$ ) under $\mathrm{N}_{2}$ atmosphere. TGA curve shows weight loss of water below $130{ }^{\circ} \mathrm{C}$ and weight loss of $N$-Ac-LCys capping ligand $\left(34 \mathrm{wt} \%\right.$ ) between $\sim 150{ }^{\circ} \mathrm{C}$ and $460{ }^{\circ} \mathrm{C}$.

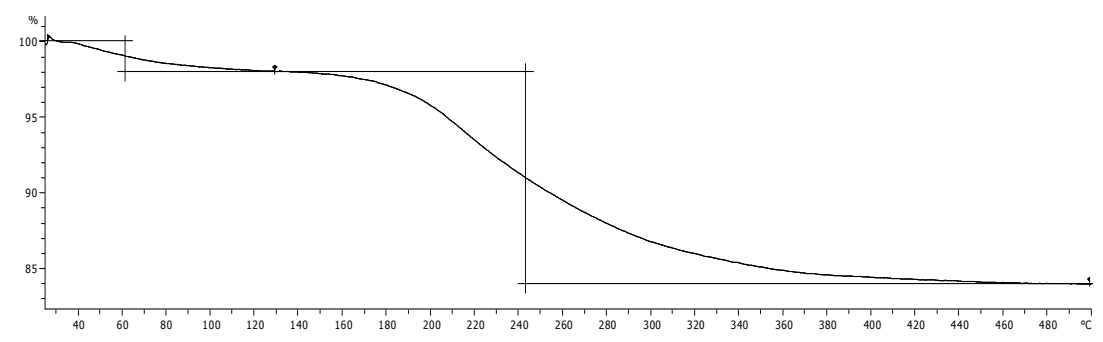

Figure S13. TGA data for $N$-Ac-L-Cys-CdSe (purified by 4 precipitations with acetone, $\varnothing_{\mathrm{CdSe}}=4.4 \mathrm{~nm}$ ) under $\mathrm{N}_{2}$ atmosphere. TGA curve shows weight loss of water below $130{ }^{\circ} \mathrm{C}$ and weight loss of $\mathrm{N}$-Ac-LCys capping ligand $\left(14.1 \mathrm{wt} \%\right.$ ) between $\sim 150{ }^{\circ} \mathrm{C}$ and $460{ }^{\circ} \mathrm{C}$.

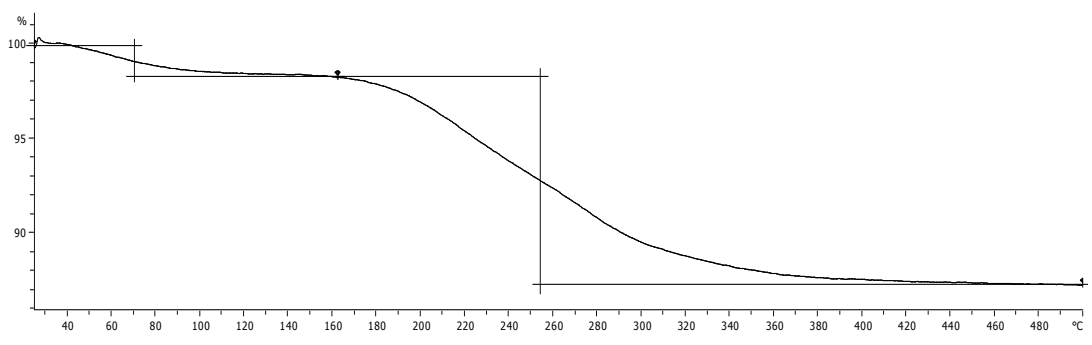

Figure S14. TGA data for $N$-Ac-L-Cys-CdSe (purified by 5 precipitations with acetone, $\varnothing_{\mathrm{CdSe}}=4.4 \mathrm{~nm}$ ) under $\mathrm{N}_{2}$ atmosphere. TGA curve shows weight loss of water below $130{ }^{\circ} \mathrm{C}$ and weight loss of $\mathrm{N}$-Ac-LCys capping ligand $\left(11.0 \mathrm{wt} \%\right.$ ) between $\sim 150{ }^{\circ} \mathrm{C}$ and $460{ }^{\circ} \mathrm{C}$. 


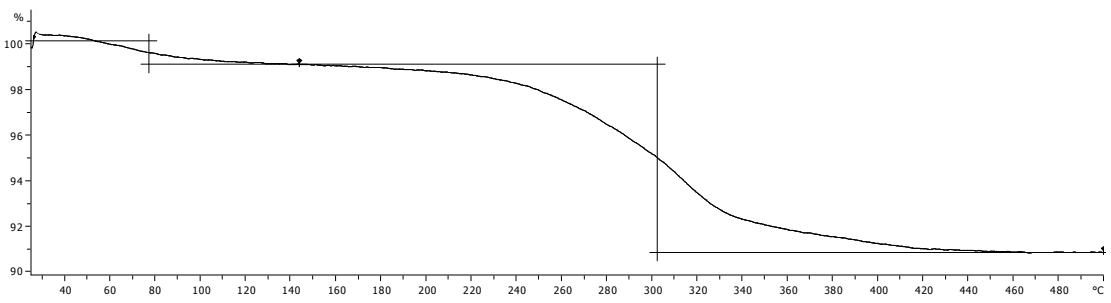

Figure S15. TGA data for $N$-Ac-L-Cys-CdSe (purified by SEC with acetone, $\varnothing_{\mathrm{CdSe}}=4.4 \mathrm{~nm}$ ). TGA curve shows weight loss of water below $\sim 130{ }^{\circ} \mathrm{C}$ and weight loss of $\mathrm{N}$-Ac-L-Cys capping ligand $(8.3 \mathrm{wt} \%)$ between $150{ }^{\circ} \mathrm{C}$ and $460{ }^{\circ} \mathrm{C}$.

Table S1: Number of ligands determined by TGA and ligand:Cd ratio for a series of precipitation of $N$-Ac-L-Cys-CdSe QDs $\left(\varnothing_{\mathrm{CdSe}}=4.4 \mathrm{~nm}\right){ }^{\mathrm{a}}$

\begin{tabular}{ccc}
\hline \# precipitations & number of ligands & ligand-to-cadmium ratio \\
\hline 2 & 2389 & 8.00 \\
3 & 746 & 2.49 \\
4 & 293 & 1.00 \\
5 & 233 & 0.78 \\
SEC & 161 & 0.54 \\
\hline
\end{tabular}

${ }^{a}$ See Methods p. S2-S3.

Table S2: CD anisotropy $g$ factors for chiral CdSe and CdS.

\begin{tabular}{|c|c|c|c|c|c|}
\hline QDs & $\varnothing^{a}$ & ligand & $\lambda_{\max } \mathrm{b}$ & $\mathrm{g}_{\mathrm{CD}}^{\mathrm{c}}$ & $\lambda_{\mathrm{CD}}{ }^{\mathrm{d}}$ \\
\hline \multirow{2}{*}{$\mathrm{CdSe}$} & \multirow{2}{*}{4.4} & L-HomoCys & 595.0 & $+1.3 \times 10^{-4}$ & 550.4 \\
\hline & & $N$-Ac-L-Cys & 595.2 & $+0.5 \times 10^{-4}$ & 584.6 \\
\hline \multirow{2}{*}{$\mathrm{CdSe}$} & \multirow{2}{*}{2.9} & L-HomoCys & 548.0 & $+0.2 \times 10^{-4}$ & 548.6 \\
\hline & & $N$-Ac-L-Cys & 546.4 & $+0.4 \times 10^{-4}$ & 531.6 \\
\hline \multirow{2}{*}{$\mathrm{CdS}$} & \multirow{2}{*}{4.3} & L-Cys & 426.8 & $+0.4 \times 10^{-4}$ & 430.0 \\
\hline & & $N$-Ac-L-Cys & 426.2 & $+0.4 \times 10^{-4}$ & 415.5 \\
\hline \multirow{2}{*}{$\mathrm{CdS}$} & \multirow{2}{*}{3.7} & L-Cys & 410.1 & $+0.8 \times 10^{-4}$ & 379.5 \\
\hline & & $N$-Ac-L-Cys & 407.5 & $+0.4 \times 10^{-4}$ & 401.0 \\
\hline
\end{tabular}

${ }^{a}$ QD diameter (in $\mathrm{nm}$ ) determined by Peng's equation ${ }^{3}$ from the absorption spectra. ${ }^{b}$ Band-edge absorption maximum (in $\mathrm{nm}$ ). ${ }^{c} \mathrm{CD}$ anisotropy $g$ factors of positive $\mathrm{CD}$ bands within CdSe band edge absorption region. ${ }^{d}$ Positive $\mathrm{CD}$ band wavelengths (in $\mathrm{nm}$ ). 

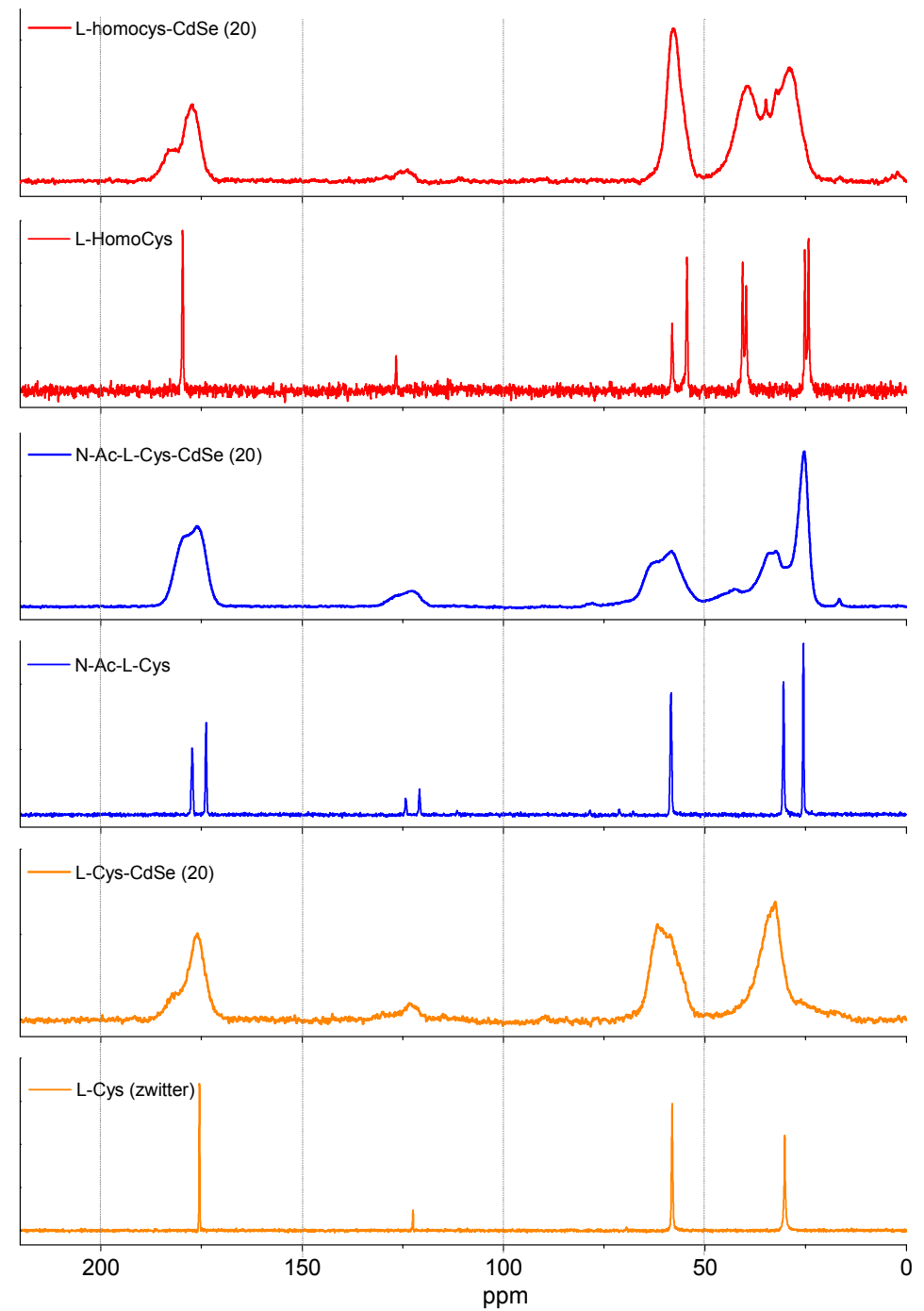

Figure S16. 1D ${ }^{13} \mathrm{C}$ MAS ssNMR spectra of (a) L-HomoCys-CdSe, (b) L-HomoCys, (c) N-Ac-L-CysCdSe, (d) $N$-Ac-L-Cys, (e) L-Cys-CdSe, and (f) L-Cys $\left(\varnothing_{\mathrm{CdSe}}=4.4 \mathrm{~nm}\right)$. All CdSe samples were purified by precipitation with acetone followed by size exclusion chromatography. MAS ssNMR spectra were acquired on a $600 \mathrm{MHz}$ Avance III Bruker NMR spectrometer at $299 \mathrm{~K}$ and $8.0 \mathrm{kHz}$ MAS frequency. 


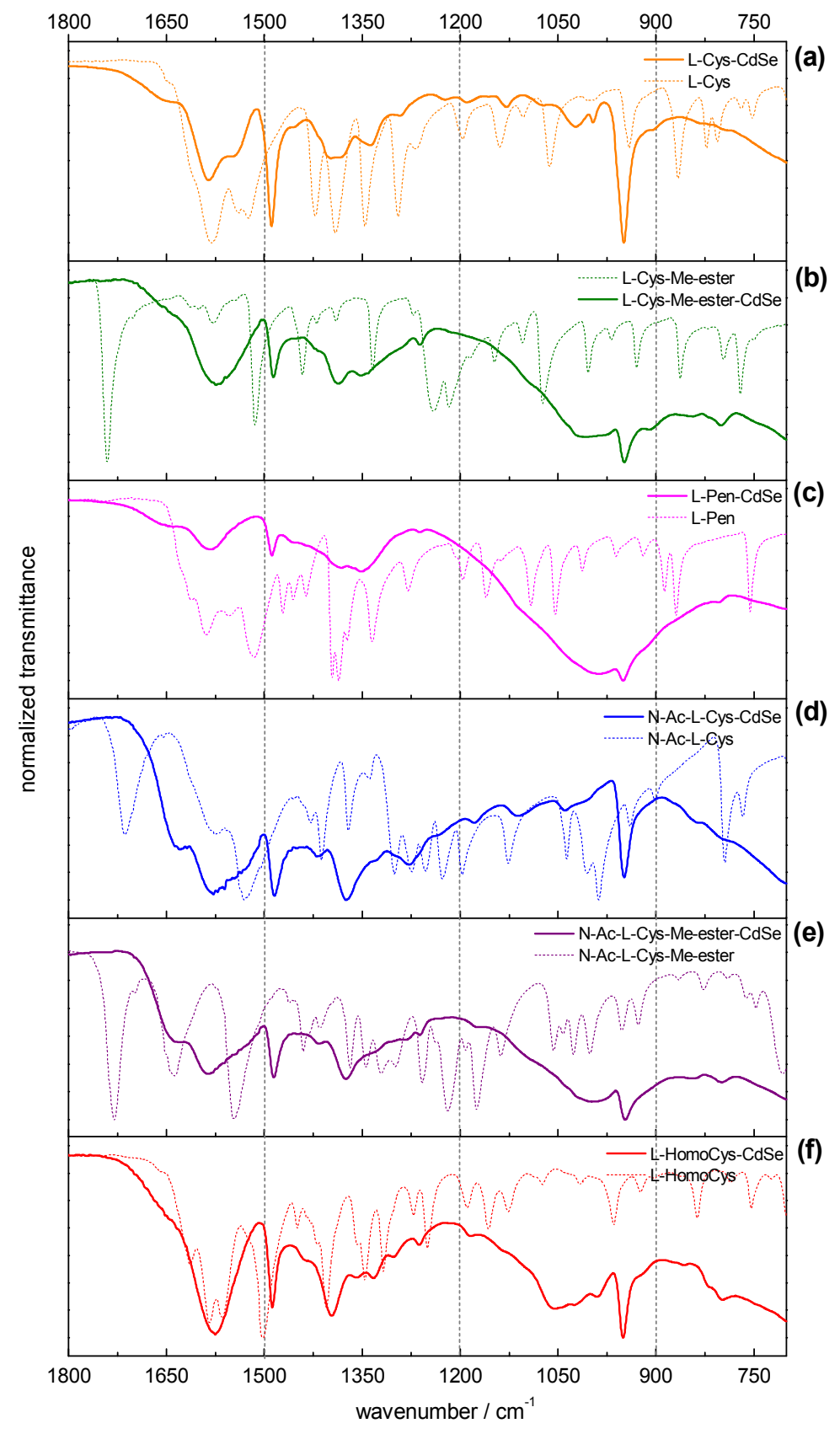

Figure S17. FTIR spectra of (a) L-Cys and L-Cys-CdSe, (b) L-Cys-Me-ester and L-Cys-Me-ester-CdSe, (c) L-Pen and L-Pen-CdSe, (d) $N$-Ac-L-Cys and $N$-Ac-L-Cys-CdSe, (e) $N$-Ac-L-Cys-Me-ester and $N$-AcL-Cys-Me-ester-CdSe, and (f) L-HomoCys and L-HomoCys-CdSe. $\left(\varnothing_{\mathrm{CdSe}}=4.4 \mathrm{~nm}\right)$. 


\section{Computational Methods.}

All computations were carried out at the density functional (DFT) and time-dependent DFT (TD-DFT) level of theory, using Gaussian 09 software. ${ }^{5}$ The geometry for the CdSe cluster was derived from the (111) surface of the cubic crystal structure as described in the main text (see also Fig. 4) and capped with hydrogen atoms to satisfy the unfilled valences (the resulting stoichiometry is $\mathrm{Cd}_{9} \mathrm{Se}_{7} \mathrm{H}_{22}$ ). The geometry optimizations were done using PBEPBE density functional ${ }^{6}$ with sbkjc-VDZ basis set/effective core potential for $\mathrm{Cd}$, Se and $\mathrm{S}$ atoms ${ }^{7}$ further augmented by two d functions (with exponents 0.4754120 and 0.2077760 ) on Se. ${ }^{1,8}$ (We denote the augmented basis set sbkjcVDZ*). For capping hydrogens and ligand atoms other than sulfur a 3-21G basis was used. The aqueous solvent was approximated by a conductorlike polarized continuum model (CPCM) with the default parameters for water. ${ }^{9}$ The CdSe cluster optimizations involved only the capping hydrogens, the $\mathrm{Cd}$ and $\mathrm{Se}$ atoms were held fixed to the experimentally determined zinc-blende (cubic) crystal geometry. ${ }^{10}$ These CdSe cluster structures were subsequently kept fixed during ligand (L-HomoCys and $N$-Ac-L-Cys) geometry optimizations.

The optimizations of both configurations with the single L-HomoCys and all models with two LHomoCys and $N$-Ac-L-Cys were optimized without any further constraints on the ligands. Optimizations of a single bound $\mathrm{N}$-Ac-L-cysteine in bi-dentate arrangements, however, had to be constrained in order to prevent convergence to the most energetically stable tri-site bound state. The initial geometries were taken from the fully optimized two $N$-Ac-L-cysteine ligand complexes, with both ligand molecules bound through Ac groups and through carboxylic groups respectively for the Ac-bound and $\mathrm{CO}_{2}{ }^{-}$bound single ligand. The structures were then optimized with the $\mathrm{S}-\mathrm{C}-\mathrm{C}-\mathrm{C}$ (for Ac bound) and $\mathrm{S}-\mathrm{C}-\mathrm{C}-\mathrm{N}$ (for $\mathrm{CO}_{2}^{-}$ bound) torsional angles fixed to the initial geometry.

Optical absorption (UV) and circular dichroism (CD) spectra were calculated with CAM-B3LYP range separated density functional, which ensures that the simulated spectra are free from artifacts due to spurious, low lying charge transfer states. ${ }^{11}$ The same basis set and solvent model were used as for the geometry optimizations. For each model compound, 200 excited states were computed. Length gauge dipolar strengths and velocity gauge rotational strengths were used, respectively, for simulations of the UV and CD spectra. The spectral contours were obtained by assigning a Gaussian line shape with full width at half maximum (FWHM) of $25 \mathrm{~nm}$ to each transition. 


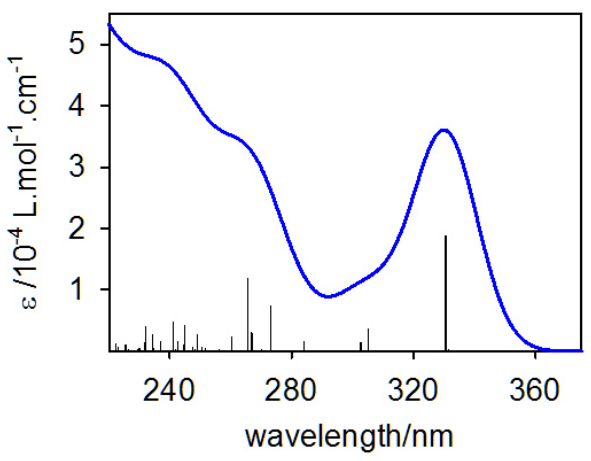

Figure S18. Simulated UV absorption spectrum for the model CdSe cluster. Vertical bars represent individual transitions (computed at CAM-B3LYP/3-21G/sbkjc-VDZ* level); the contour was obtained using Gaussian line shape with $25 \mathrm{~nm}$ half-width. The blue shift of the computed spectrum with respect to experiment is expected due to the much smaller size of the model cluster; however, the pronounced excitonic band, along with the partially resolved shoulder near $270 \mathrm{~nm}$ on the sloping absorption continuum to the blue reflected the characteristic experimental spectra (Figure $1 \mathrm{f}$ of the main text).

(a)

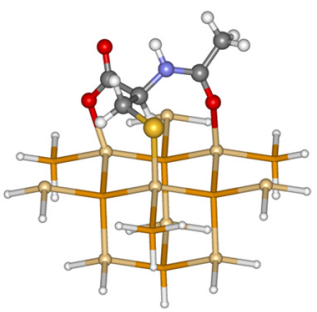

(b)
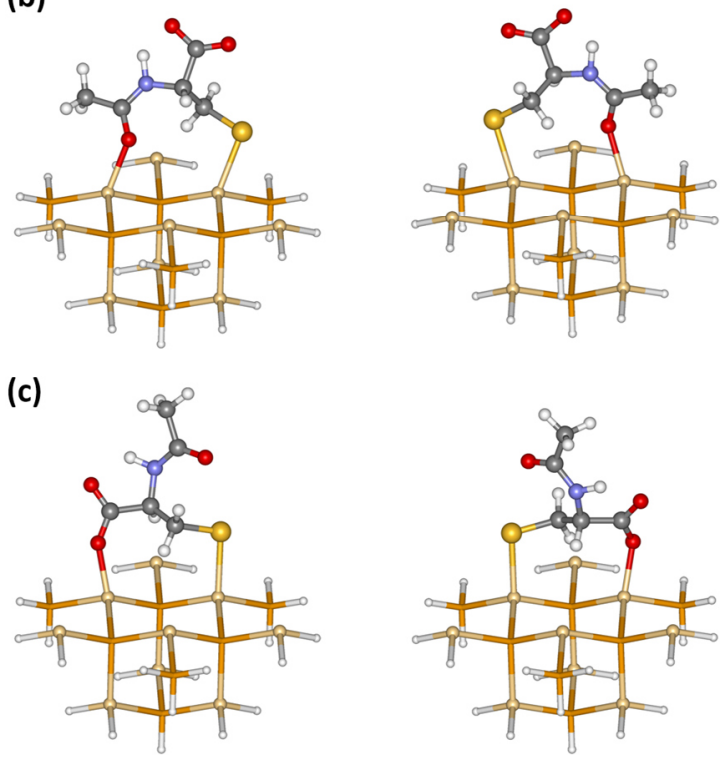

Figure S19. Optimized structures of $N$-Ac-L-Cys ligand on the surface of a model QD fragment. The optimizations were carried out at PBEPBE/3-21G/sbkjc-VDZ* level. (a) Fully optimized tri-site bound $N$ Ac-L-Cys. (b) Two-site thiol and acetyl bound and (c) two-site thiol and carboxylate bound structures for enantiomer \#1 (left) and \#2 (right) optimized with the S-C-C-C torsional angles constrained in order to prevent convergence to the triply bound state. The relative energies are listed in Table S3. 
Table S3: Relative energies in kcal.mol ${ }^{-1}$ of $\mathrm{CdSe}$ complexes with a single ligand ${ }^{a}$

\begin{tabular}{|c|c|c|c|}
\hline ligand & binding $^{b}$ & $\# 1^{c}$ & $\# 2$ \\
\hline L-homocysteine & $\mathrm{S} / \mathrm{COO}$ & $\mathbf{0 . 0 ^ { d }}$ & 1.4 \\
\hline \multirow[t]{3}{*}{$N$-acetyl-L-cysteine } & $\mathrm{S} / \mathrm{Ac} / \mathrm{COO}$ & \multicolumn{2}{|c|}{0.0} \\
\hline & $\mathrm{S} / \mathrm{Ac}$ & 27.1 & 24.0 \\
\hline & $\mathrm{S} / \mathrm{COO}$ & 22.5 & 23.4 \\
\hline
\end{tabular}

${ }^{a}$ Relative to the most stable configuration. Electronic energies (including PCM) solvation are reported. ZPE and other nuclear energy terms are not meaningful due to constraints.

${ }^{b}$ The S, Ac, and COO denotes binding to the model CdSe surface by the thiol, acetyl or carboxylate groups respectively.

${ }^{c}$ Numbered according to Figure 4 of the main text and Figure S19.

${ }^{d}$ Values highlighted in bold correspond to the geometries for which the simulated CD spectra are shown in Figure 5 of the main text.

Table S4: Relative energies in kcal.mol ${ }^{-1}$ of CdSe complexes with two ligands ${ }^{a}$

\begin{tabular}{llcccccc}
\hline ligand & binding $^{b}$ & $\mathbf{\# 1}^{c}$ & $\mathbf{\# 2}$ & $\mathbf{\# 3}$ & $\mathbf{\# 5}$ & $\mathbf{\# 6}$ & $\# \mathbf{7 7}$ \\
\hline L-homocysteine & $\mathrm{COO} / \mathrm{COO}$ & 8.0 & 9.3 & 0.0 & 16.7 & $\mathbf{1 4 . 1}^{f}$ & 7.8 \\
\hline \multirow{2}{*}{$N$-acetyl-L-cysteine } & $\mathrm{Ac} / \mathrm{Ac}$ & 41.6 & 42.1 & 44.6 & 45.7 & 36.3 & 36.0 \\
& $\mathrm{Ac} / \mathrm{COO}^{d}$ & 18.7 & 18.0 & 1.9 & 14.4 & 11.6 & 24.5 \\
& $\mathrm{COO} / \mathrm{Ac}^{e}$ & 24.6 & $\mathbf{2 2 . 8}$ & 23.0 & 20.8 & 10.3 & 15.8 \\
& $\mathrm{COO} / \mathrm{COO}$ & $\mathbf{5 . 6}$ & 2.7 & 8.5 & 0.0 & 9.2 & 0.1 \\
\hline
\end{tabular}

${ }^{a}$ Relative to the most stable configuration. Electronic energies (including PCM) solvation are reported. ZPE and other nuclear energy terms are not meaningful due to constraints.

${ }^{b}$ The S, Ac, and COO denotes binding to the model CdSe surface by the thiol, acetyl or carboxylate groups respectively.

${ }^{c}$ Numbered according to Figure 6e of the main text.

${ }^{d}$ Ac group binding on "inner" Cd atoms (with Se neighbors) of the model CdSe fragment, COO group on "outer" Cd atoms (with hydrogen capping).

e COO group binding on "inner" Cd atoms (with Se neighbors) of the model CdSe fragment, Ac group on "outer" Cd atoms (with hydrogen capping).

${ }^{f}$ Values highlighted in bold correspond to the geometries for which the simulated CD spectra are shown in Figure 6 of the main text. 


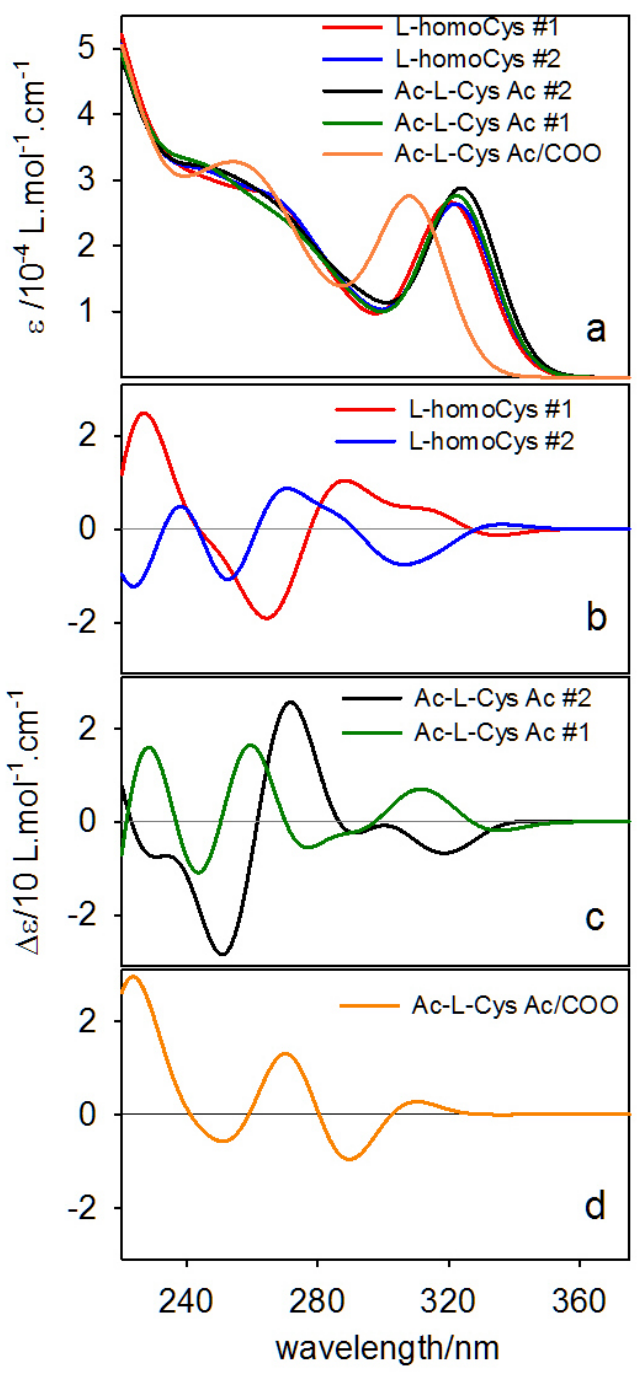

Figure S20. Simulated UV and CD spectra for model CdSe QD with a single L-HomoCys and $N$-AcL-Cys ligand doubly-bound in two opposing arrangements, as depicted in Figure 4 of the main text, and for the triply-bound N-Ac-L-Cys. (a) UV absorption spectra, (b) CD spectra for CdSe with LHomoCys ligands, (c) CD spectra of CdSe with $N$-Ac-L-Cys ligands, (d) CD spectra of CdSe with the $N$-Ac-L-Cys ligand bound through thiol, acetyl and carboxylate groups. 
\#1
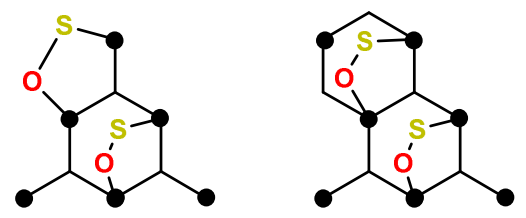

\#1
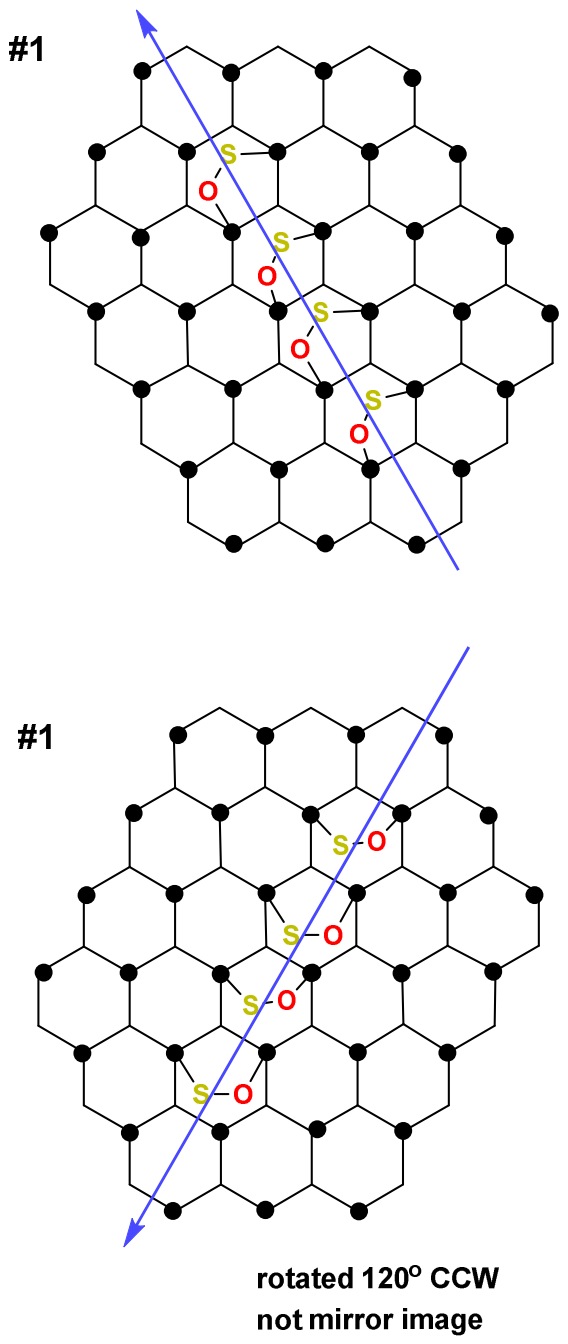

\#3
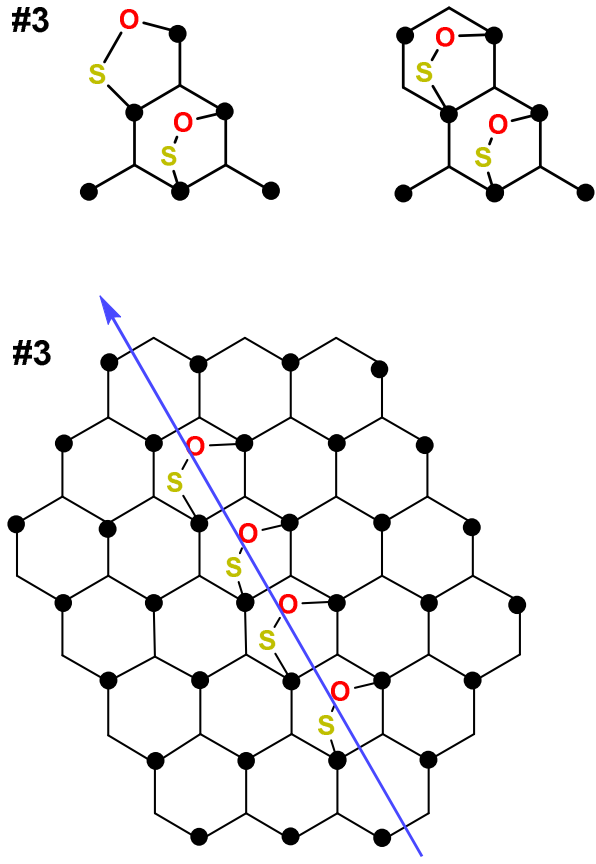

\#1

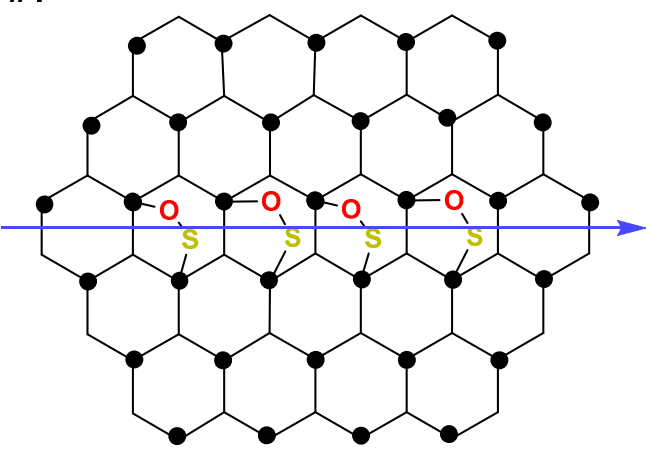

rotated $120^{\circ} \mathrm{CW}$

not mirror image

Figure S21. Schematic illustrations of the bi-dentate binding arrangements \#1 and \#3 on the model QD surfaces, constructed as repeats of the elementary CdSe fragment used as a minimal model. The $\mathrm{Cd}$ atoms are represented by black dots. 
\#2

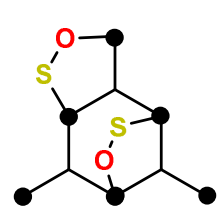

\#2

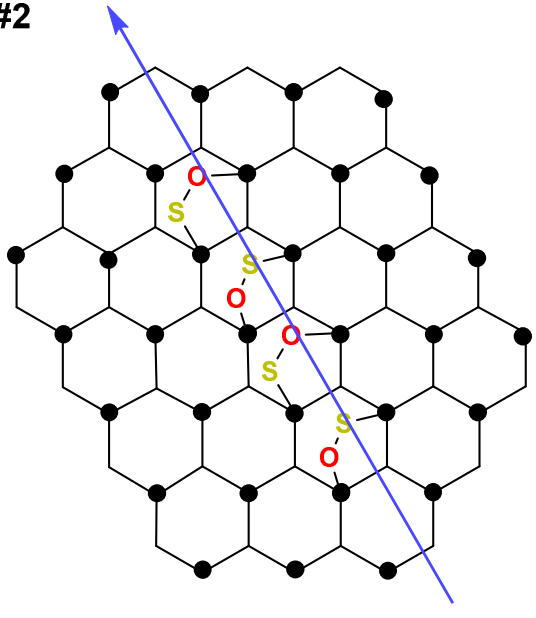

\#4

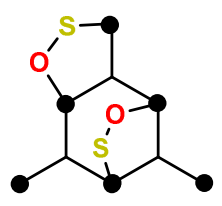

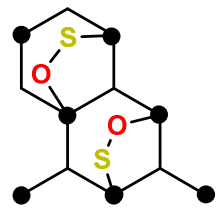

\#4

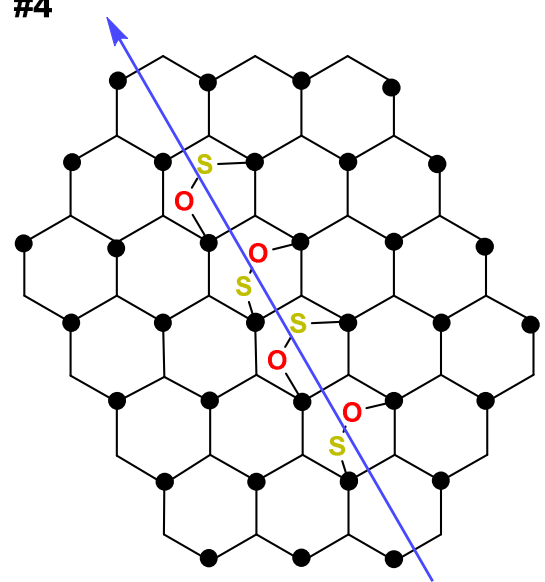

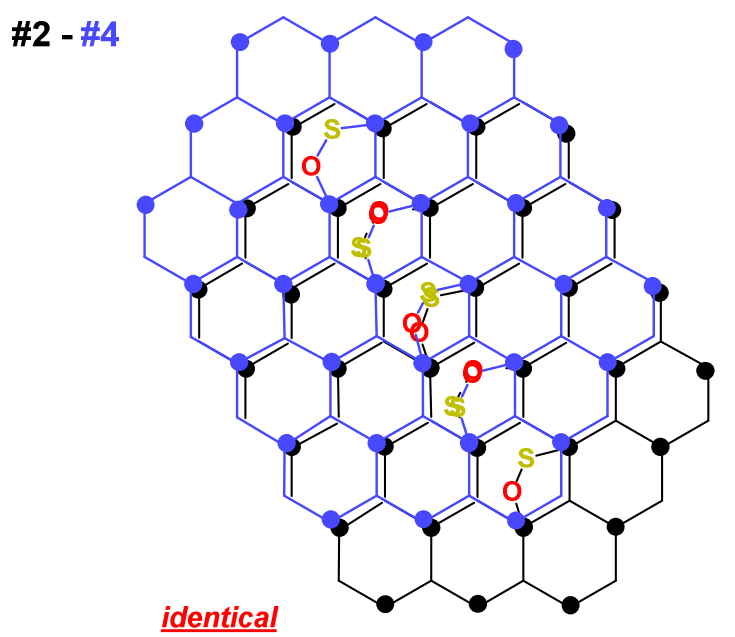

Figure S22. Schematic illustrations of the bi-dentate binding arrangements \#2 and \#4, demonstrating that they yield identical structures. The $\mathrm{Cd}$ atoms are represented by black dots. 
\#5<smiles>C[C@H]1[C@@H](C)[C@@H]2O[C@H]1[C@H]1OSC[C@@H]21</smiles>

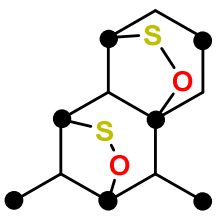

\#5

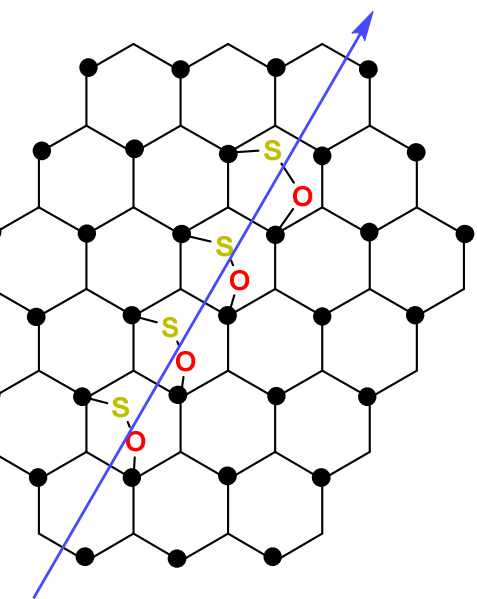

\#5

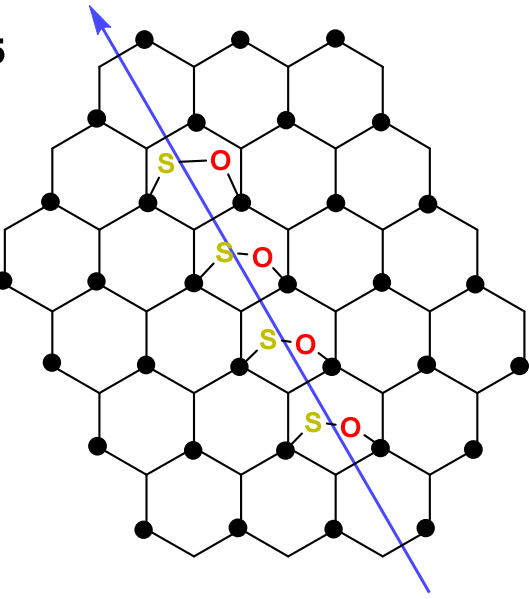

rotated $60^{\circ} \mathrm{CCW}$

not mirror image
\#7<smiles>C[C@H]1[C@H](C)[C@H]2[C@@H]([C@@H]3COOS3)O[C@H]12</smiles>

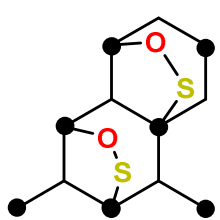

\#7

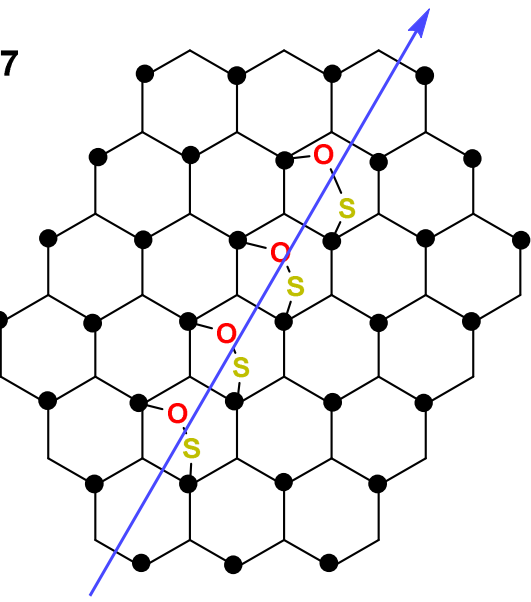

\#5

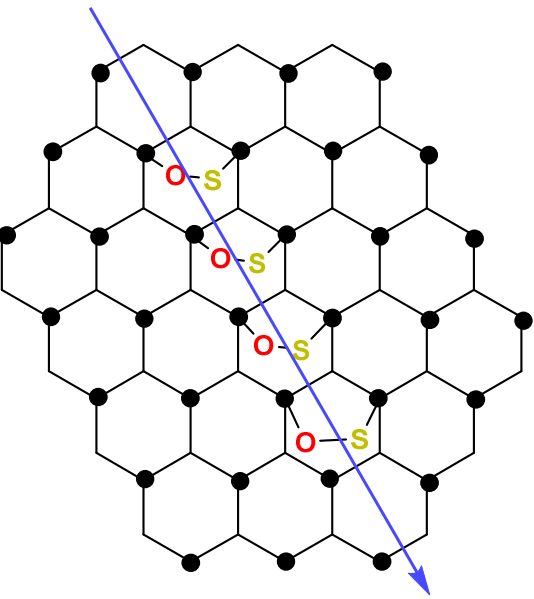

rotated $120^{\circ} \mathrm{CW}$

not mirror image

Figure S23. Schematic illustrations of the bi-dentate binding arrangements \#5 and \#7 on the model QD surfaces, constructed as repeats of the elementary CdSe fragment used as a minimal model. The Cd atoms are represented by black dots. 
\#6<smiles>C[C@@H]1[C@@H]2O[C@H]([C@@H]3CSO[C@@H]32)[C@@H]1C</smiles>

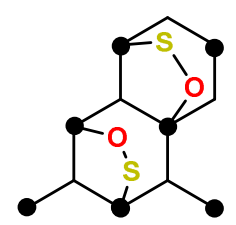

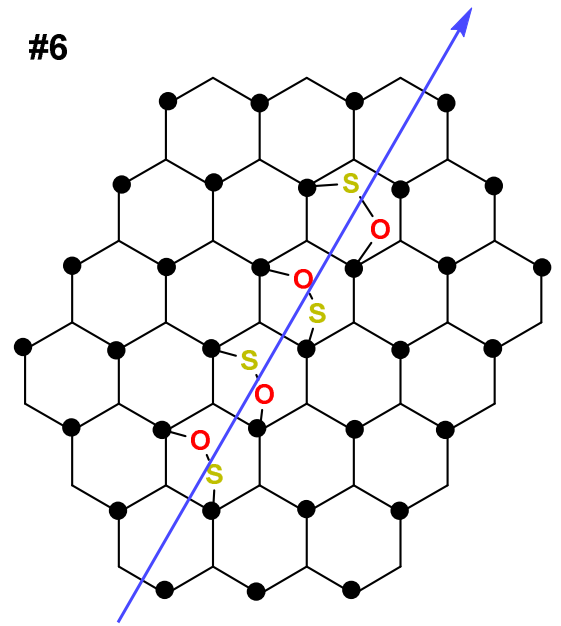

\#8

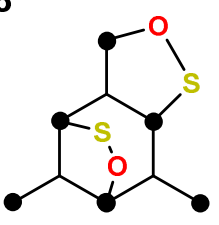

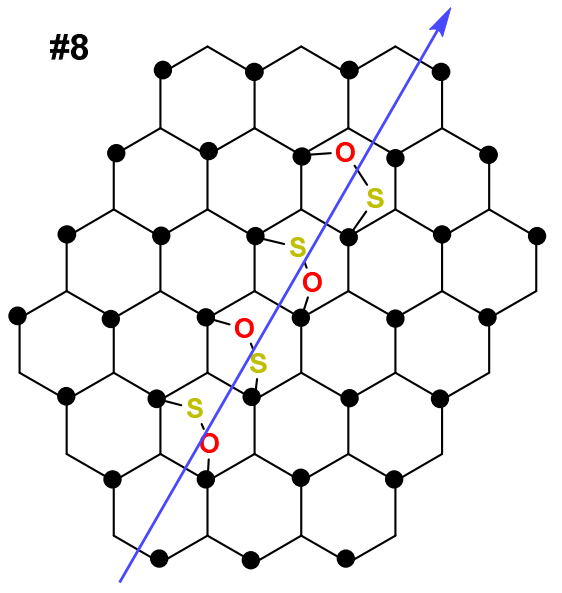

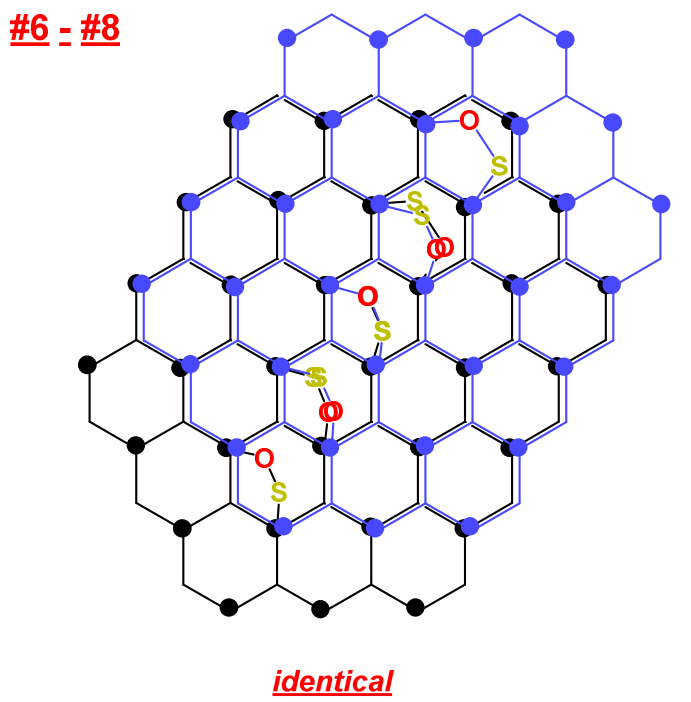

Figure S24 Schematic illustrations of the bi-dentate binding arrangements \#6 and \#8, demonstrating that they yield identical structures. The $\mathrm{Cd}$ atoms are represented by black dots. 

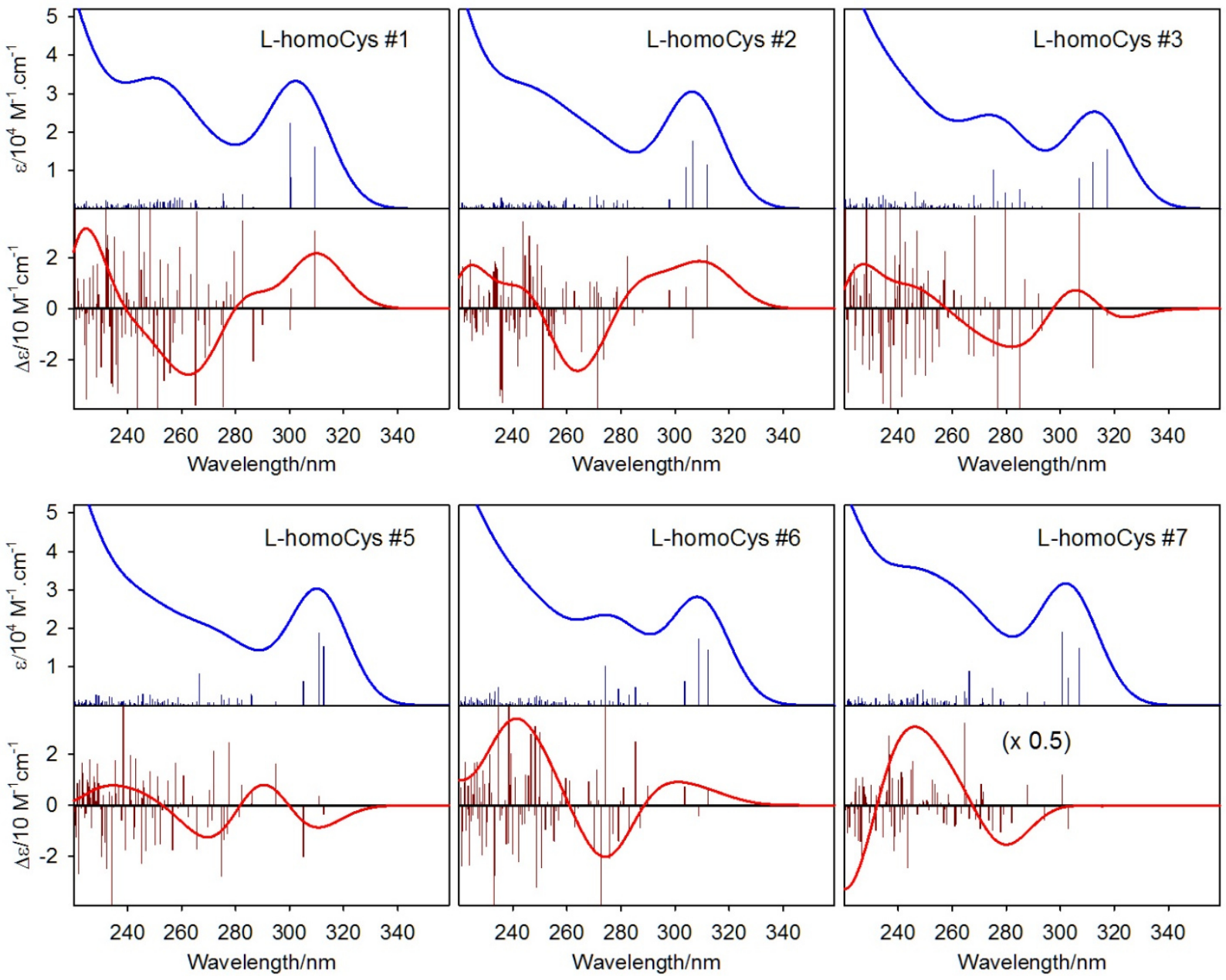

Figure S25. Simulated UV and CD spectra for model CdSe QD with two L-HomoCys ligands. The numbering of individual conurations corresponds to Figure 6 of the main text (also see Figures S21 S24). The individual transitions are represented by vertical lines. The spectral contours were obtained by assigning a Gaussian profile with $25 \mathrm{~nm}$ half-width to each transition. 

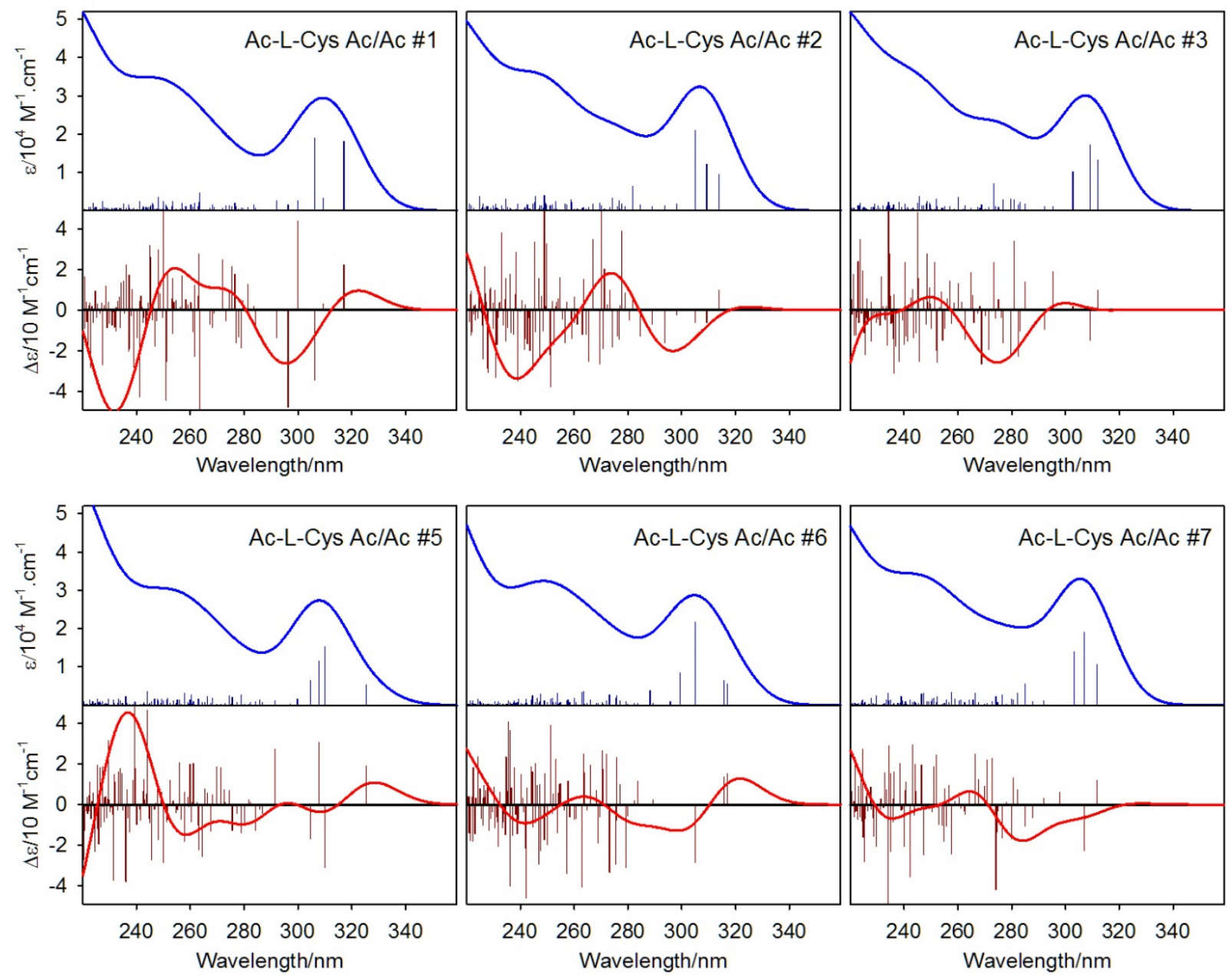

Figure S26. Simulated UV and CD spectra for model CdSe QD with two N-Ac-L-Cys ligands, both bound to the CdSe surface with Ac groups. The numbering of individual configurations corresponds to Figure 6 of the main text (also see Figures S21 - S24). The individual transitions are represented by vertical lines. The spectral contours were obtained by assigning a Gaussian profile with $25 \mathrm{~nm}$ half-width to each transition. 

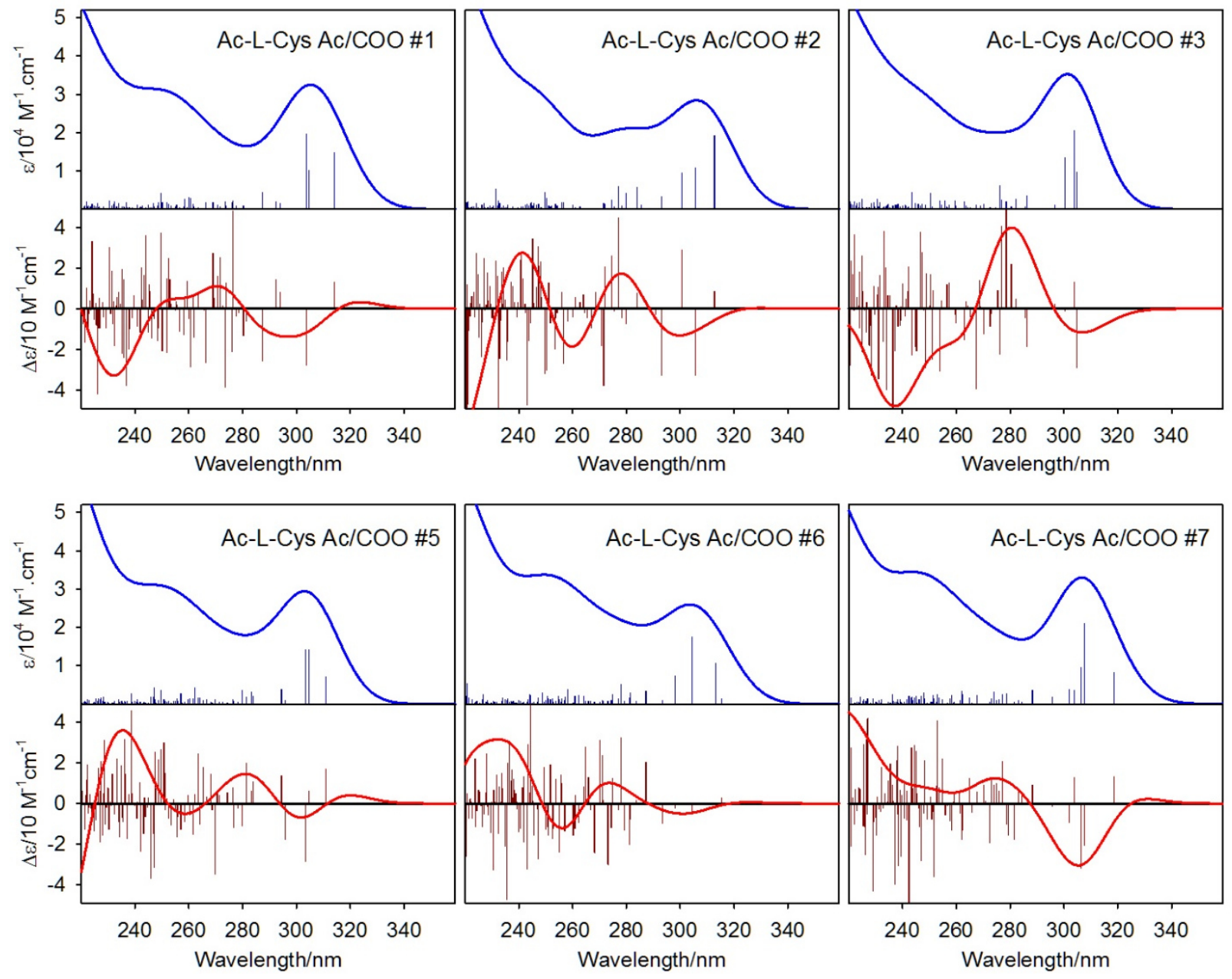

Figure S27. Simulated UV and CD spectra for model CdSe QD with two $N$-Ac-L-Cys ligands bound to the CdSe surface through the Ac (to $\mathrm{Cd}$ atoms of the inner CdSe hexagon) and $\mathrm{COO}^{-}$(to outer, hydrogen capped $\mathrm{Cd}$ atoms) groups. The numbering of individual configurations corresponds to Figure 6 of the main text (also see Figures S21 - S24). The individual transitions are represented by vertical lines. The spectral contours were obtained by assigning a Gaussian profile with $25 \mathrm{~nm}$ half-width to each transition. 

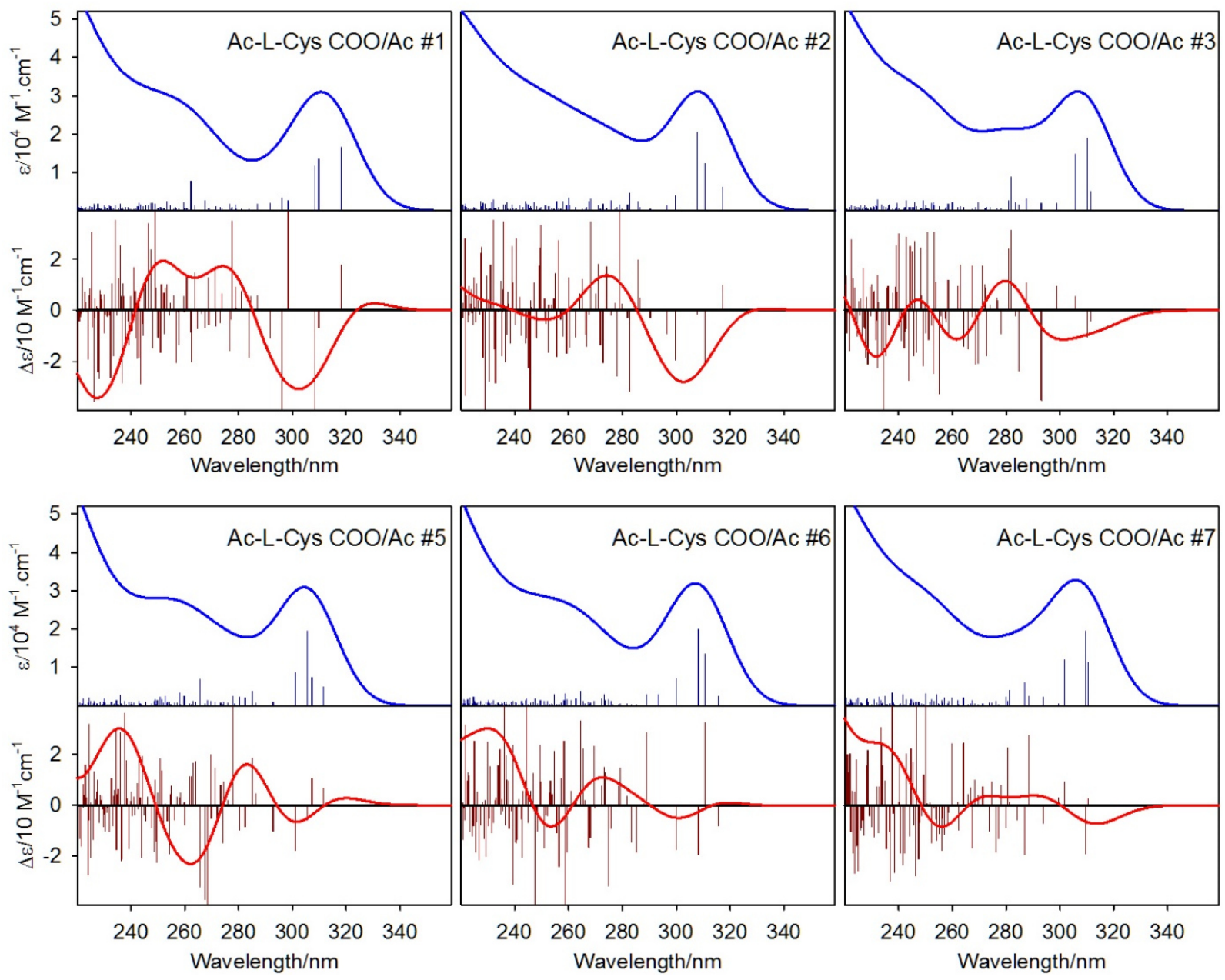

Figure S28. Simulated UV and CD spectra for model CdSe QD with two $N$-Ac-L-Cys ligands bound to the CdSe surface through the $\mathrm{COO}^{-}$(to $\mathrm{Cd}$ atoms of the inner CdSe hexagon) and Ac (to outer, hydrogen capped Cd atoms) groups. The numbering of individual configurations corresponds to Figure 6 of the main text (also see Figures S21 - S24). The individual transitions are represented by vertical lines. The spectral contours were obtained by assigning a Gaussian profile with $25 \mathrm{~nm}$ half-width to each transition. 

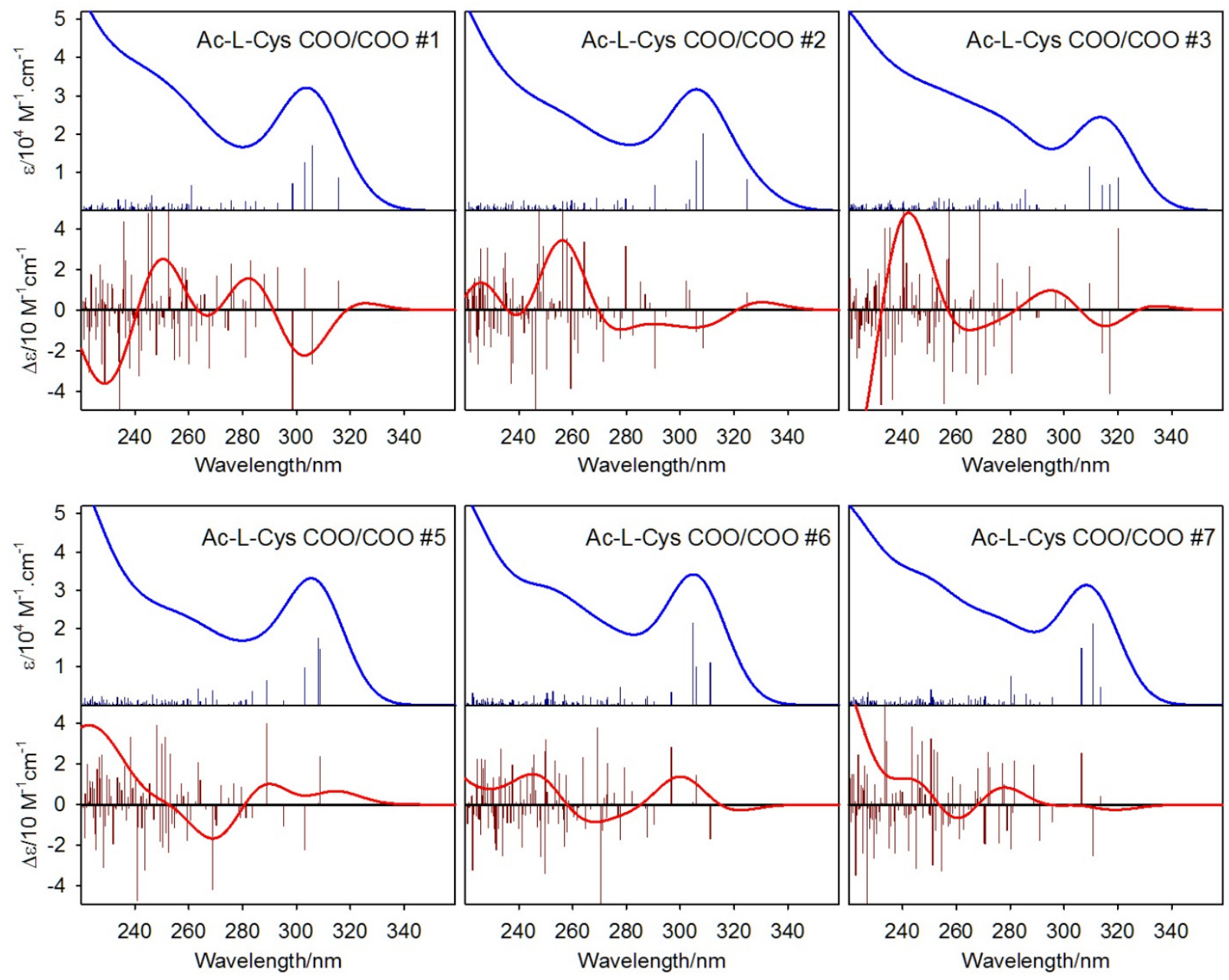

Figure S29. Simulated UV and CD spectra for model CdSe QD with two $N$-Ac-L-Cys ligands, both bound to the CdSe surface with $\mathrm{COO}^{-}$groups. The numbering of individual configurations corresponds to Figure 6 of the main text (also see Figures S21 - S24). The individual transitions are represented by vertical lines. The spectral contours were obtained by assigning a Gaussian profile with $25 \mathrm{~nm}$ half-width to each transition. 


\section{Coordinates of the $\mathrm{Cd}_{9} \mathrm{Se}_{7} \mathrm{H}_{22}$ cluster:}

$\begin{array}{rrrr}\mathrm{H} & 4.270076 & 4.194419 & -0.648136 \\ \mathrm{H} & -4.270076 & 4.194419 & -0.648136 \\ \mathrm{H} & -5.767511 & 1.600785 & -0.648136 \\ \mathrm{H} & -1.497435 & -5.795203 & -0.648136 \\ \mathrm{H} & 1.497435 & -5.795203 & -0.648136 \\ \mathrm{H} & 5.767511 & 1.600785 & -0.648136 \\ \mathrm{H} & 1.219068 & 5.634519 & -0.885048 \\ \mathrm{H} & -1.219068 & 5.634519 & -0.885048 \\ \mathrm{H} & -5.489171 & -1.761516 & -0.885048 \\ \mathrm{H} & -4.270103 & -3.873004 & -0.885048 \\ \mathrm{H} & 4.270103 & -3.873004 & -0.885048 \\ \mathrm{H} & 5.489171 & -1.761516 & -0.885048 \\ \mathrm{H} & 0.000000 & 4.930974 & 1.104937 \\ \mathrm{H} & -4.270349 & -2.465487 & 1.104937 \\ \mathrm{H} & 4.270349 & -2.465487 & 1.104937 \\ \mathrm{H} & 2.135267 & 2.962165 & 2.838916 \\ \mathrm{H} & -2.135267 & 2.962165 & 2.838916 \\ \mathrm{H} & -3.632944 & 0.368112 & 2.838916 \\ \mathrm{H} & -1.497677 & -3.330278 & 2.838916 \\ \mathrm{H} & 1.497677 & -3.330278 & 2.838916 \\ \mathrm{H} & 3.632944 & 0.368112 & 2.838916 \\ \mathrm{H} & 0.000000 & 0.000000 & 4.585988 \\ \mathrm{Se} & 0.000000 & 4.930959 & -0.388063 \\ \mathrm{Se} & -4.270335 & -2.465479 & -0.388063 \\ \mathrm{Se} & 4.270335 & -2.465479 & -0.388063 \\ \mathrm{Se} & -2.135673 & 1.233031 & -0.388065 \\ \mathrm{Se} & 0.000000 & -2.466063 & -0.388065 \\ \mathrm{Se} & 2.135673 & 1.233031 & -0.388065 \\ \mathrm{Se} & 0.000000 & 0.000000 & 3.098988 \\ \mathrm{Cd} & -4.270409 & 2.465522 & -1.259117 \\ \mathrm{Cd} & 0.000000 & -4.931044 & -1.259117 \\ \mathrm{Cd} & 4.270409 & 2.465522 & -1.259117 \\ \mathrm{Cd} & 0.000000 & 2.465485 & -1.259038 \\ \mathrm{Cd} & -2.135721 & 1.233059 & 2.226935 \\ \mathrm{Cd} & -2.135173 & -1.232743 & -1.259038 \\ \mathrm{Cd} & 0.000000 & -2.466118 & 2.226935 \\ \mathrm{Cd} & 2.135173 & -1.232743 & -1.259038 \\ \mathrm{Cd} & 2.135721 & 1.233059 & 2.226935\end{array}$




\section{$\underline{\text { References }}$}

1. Tohgha, U.; Deol, K. K.; Porter, A. G.; Bartko, S. G.; Choi, J. K.; Leonard, B. M.; Varga, K.; Kubelka, J.; Muller, G.; Balaz, M. Ligand Induced Circular Dichroism and Circularly Polarized Luminescence in Cdse Quantum Dots. ACS Nano 2013, 7, 11094-11102.

2. Tohgha, U.; Varga, K.; Balaz, M. Achiral Cdse Quantum Dots Exhibit Optical Activity in the Visible Region Upon Post-Synthetic Ligand Exchange with D- or L-Cysteine. Chem. Commun. 2013, 49, 1844-1846.

3. Yu, W. W.; Qu, L.; Guo, W.; Peng, X. Experimental Determination of the Extinction Coefficient of Cdte, Cdse, and Cds Nanocrystals. Chem. Mater. 2003, 15, 2854-2860.

4. Morcombe, C. R.; Zilm, K. W. Chemical Shift Referencing in Mas Solid State Nmr. J. Magn. Reson. 2003, 162, 479-486.

5. Frisch, M. J.; Trucks, G. W.; Schlegel, H. B.; Scuseria, G. E.; Robb, M. A.; Cheeseman, J. R.; Scalmani, G.; Barone, V.; Mennucci, B.; Petersson, G. A.; Nakatsuji, H.; Caricato, M. L., X.; ; Hratchian, H. P.; Izmaylov, A. F.; Bloino, J.; Zheng, G.; Sonnenberg, J. L.; Hada, M.; Ehara, M.; Toyota, K., et al. Gaussian 09, Gaussian, Inc.: Wallingford CT, 2009.

6. Perdew, J. P.; Burke, K.; Ernzerhof, M. Generalized Gradient Approximation Made Simple. Phys. Rev. Lett. 1996, 77, 3865-3868.

7. Stevens, W. J.; Krauss, M.; Basch, H.; Jasien, P. G. Relativistic Compact Effective Potentials and Efficient, Shared-Exponent Basis-Sets for the 3rd-Row, 4th-Row, and 5th-Row Atoms. Can. J. Chem. 1992, 70, 612-630.

8. Azpiroz, J. M.; Matxain, J. M.; Infante, I.; Lopez, X.; Ugalde, J. M. A Dft/Tddft Study on the Optoelectronic Properties of the Amine-Capped Magic (Cdse)13 Nanocluster. Phys. Chem. Chem. Phys. 2013, 15, 10996-11005.

9. Cossi, M.; Rega, N.; Scalmani, G.; Barone, V. Energies, Structures, and Electronic Properties of Molecules in Solution with the C-Pcm Solvation Model. J. Comput. Chem. 2003, 24, 669-681.

10. Kulakov, M. P.; Balyakina, I. V.; Kolesnikov, N. N. Phase-Diagram and Crystallization in the System Cdse-Znse Inorg. Mater. (Engl. Transl.) 1989, 25, 1386-1389.

11. Magyar, R. J.; Tretiak, S. Dependence of Spurious Charge-Transfer Excited States on Orbital Exchange in Tddft: Large Molecules and Clusters. J. Chem. Theory Comput. 2007, 3, 976-987. 